\title{
Model-based decomposition of dual-pol SAR data: application to Sentinel-1
}

\author{
Lucio Mascolo, Member, IEEE, Shane R. Cloude, Fellow, IEEE, and Juan M. Lopez-Sanchez, Senior \\ Member, IEEE
}

\begin{abstract}
In this study, we advance a new family of modelbased decompositions adapted for dual-pol synthetic aperture radar data. These are formulated using the Stokes vector formalism, coupled to mappings from full quad-pol decomposition theory. A generalized model-based decomposition is developed, which allows separation of an arbitrary Stokes vector into partially polarized and polarized wave components. We employ the widely used random dipole cloud as a volume model but, in general, non-dipole options can be used. The cross-polarized phase $\delta$, and the $\alpha$ angle, which is a function of the ratio between wave components, measure the transformation of polarization state on reflection. We apply the decomposition to dual-pol data provided by Sentinel-1 covering different scenarios, such as agricultural, forest, urban and glacial land-ice. We show that the polarized term of received polarization state is not usually the same as the transmitted one, and can therefore be used for key applications, e.g., classification and geo-physical parameter estimation. We show that, for vegetated terrain, depolarization is not the only influencing factor to Sentinel-1 backscattered intensities and, in the case of vertical crops (e.g., rice), this allows the crop orientation effects to be decoupled from volume scattering in the canopy. We demonstrate that coherent dualpol systems show strong phase signatures over glaciers, where the polarized contribution significantly affects the backscattered state, resulting in elliptical polarization on receive. This is a key result for Sentinel-1, for which dual-pol phase analysis coupled to dense time series offer great opportunities for land-ice monitoring.
\end{abstract}

Index Terms-Sentinel-1, polarimetric decompositions, wave polarimetry, land-cover, land-ice.

\section{INTRODUCTION}

Polarimetric synthetic aperture radar (POLSAR) is an important space-borne imaging technology, which has been exploited successfully in many different application domains of Earth Observation [1]. One key advantage of fully polarimetric systems (where the complete $2 \times 2$ complex scattering matrix is measured for each pixel [2]) is a clearer physical interpretation of the measured radar data, since POLSAR provides maximum flexibility and adaptability for retrieval of geo-physical parameters from the imaged scene. One of the most widely used approaches for POLSAR data analysis is based on target decomposition (TD) techniques [3]. TD

This work was funded by the Spanish Ministry of Science and Innovation, the State Agency of Research (AEI) and the European Funds for Regional Development (EFRD) under Projects TEC2017-85244-C2-1-P and PID2020$117303 \mathrm{~GB}-\mathrm{C} 22$, and by the University of Alicante under grant VIGROB-114.

L. Mascolo, J.M. Lopez-Sanchez are with the Institute for Computer Research (IUII), University of Alicante, Alicante, Spain e-mail: \{lucio.mascolo,juanma.lopez\}@ua.es

S.R. Cloude is with AEL Consultants, Cupar KY15 5AA, U.K. email:aelc@mac.com aims at providing a physical interpretation of the scene by expressing the radar response as a combination of responses from canonical scattering elements [4]. Among TD techniques, model-based decompositions are especially appealing [1], [2], [4], [5] because the components are directly related to physical elements present in a scene (e.g., surface scattering from soil, volume scattering from vegetation canopies, etc.). Consequently, many model- based decomposition techniques have been proposed, starting from the original ideas of Freeman and Durden [6] via various levels of adaptation and expansion [7][9] towards what is still an active field of research [10], [11].

All such decompositions are designed to be used only with quad-pol data, like that provided by a few operational satellite systems such as the Canadian Space Agency (CSA) Radarsat2 (RS2) and Japanese Space Agency (JAXA), ALOS-2. On the contrary, many more operational space-borne systems provide only dual-pol data (where only a column of the scattering matrix is measured for each pixel). Here we emphasize the importance of providing coherent dual-pol data from such sensors, where the relative phase difference between channels is measured along with radiometrically calibrated backscatter. Such data is provided, for example, by a constellation operated by the European Space Agency (ESA) via their Sentinel1 (S1) satellites. To date, little effort has been devoted to formulating decompositions adapted to such coherent dualpol data. However, S1 data are freely accessible globally and offer a short revisit time (6 days in Europe, 12 days elsewhere). Therefore, they are very well suited for implementing operational monitoring services based on a reliable and consistent measurement schedule. In this context, there exists an opportunity to investigate the potential for modelbased decompositions for dual-pol data, such as S1, with a view to improving the physical interpretation of such data. We consider one such family of model-based decompositions in this paper and demonstrate its application to a wide variety of applications taken from the S1 data base.

Before considering details of the proposed decomposition, it is important to clearly state that, due to the limited observation space (dual-pol instead of quad-pol), the possibilities for decomposition of dual-pol data are inherently limited. Nonetheless, we shall show that some elements of physical modelling can be used to better exploit information in such systems, although dual-pol limitations result in a strictly different interpretation of many familiar decomposition parameters. For example, we will define an alpha angle, familiar from many full decomposition approaches [2], [5], as a ratio of wave components, not as a ratio of true scattering coef- 
ficients. Consequently, the selective availability of different combinations of dual polarimetric channels, e.g., VH-VV or $\mathrm{HH}-\mathrm{HV}$, will have an impact on the interpretation of the decomposition outputs, as well as on the identification or measurement of potentially observed phenomena taking place at the scene. In summary, dual-pol data are always subject to strong ambiguities produced by the lack of the remaining polarimetric channels. These aspects are carefully addressed in the manuscript, and, despite the clear limitations, we show that the proposed dual-pol decompositions can be used to extract important underlying physical information, provided that the correct interpretation of parameters is used.

In Section II we first formulate a general approach to dualpol decompositions, using a wave Stokes vector formulation coupled to mappings from full quad-pol decomposition theory. In Section III we then apply these new decompositions to four important case studies using dual-pol S1 data, namely urban areas monitoring, agriculture (rice growth monitoring), land-cover monitoring and glacial land-ice monitoring. In Section IV we discuss and analyze the results obtained and, in Section V, draw conclusions concerning the potential for widespread application of TD techniques to dual-pol systems.

\section{Formulation OF DUAL-Pol DECOMPOSITIONS}

Dual-pol systems employ a single transmitter polarization state with a dual channel coherent receiver, measuring both horizontal $(\mathrm{H})$ and vertical $(\mathrm{V})$ components of the scattered wave. Following calibration and SAR processing, we then obtain a 2-element complex vector $\underline{E}$ for each pixel in the image. This single-look complex (SLC) data is then multilooked and speckle filtered before use in applications. Under usual assumptions of ergodicity and stationarity [12], such filtering leads to the generation of an average $2 \times 2$ wave coherency matrix $\mathrm{C} 2$ for each pixel, as shown in (1), where averaging is generally performed over a local spatial window.

$$
\mathrm{C} 2=\left\langle\left[\begin{array}{l}
E_{H T} \\
E_{V T}
\end{array}\right]\left[\begin{array}{ll}
E_{H T}^{*} & E_{V T}^{*}
\end{array}\right]\right\rangle=\left[\begin{array}{ll}
c_{11} & c_{12} \\
c_{12}^{*} & c_{22}
\end{array}\right]
$$

Note that the subscript $T$ in (1) is the transmit polarization state, which for $\mathrm{S} 1$ can be either linear V or H. The Stokes vector of the scattered wave $\underline{s}$ is then fully determined from C2, as shown in (2), with $\underline{s}_{H}$ and $\underline{s}_{V}$ being important examples.

$$
\underline{s}=\left[\begin{array}{l}
s_{1} \\
s_{2} \\
s_{3} \\
s_{4}
\end{array}\right]=\left[\begin{array}{l}
c_{11}+c_{22} \\
c_{11}-c_{22} \\
2 \operatorname{Re}\left(c_{12}\right) \\
2 \operatorname{Im}\left(c_{12}\right)
\end{array}\right] \rightarrow \underline{s}_{H}=\left[\begin{array}{l}
1 \\
1 \\
0 \\
0
\end{array}\right], \underline{s}_{V}=\left[\begin{array}{c}
1 \\
-1 \\
0 \\
0
\end{array}\right]
$$

Note that here we employ the backscatter alignment (BSA) coordinate system [5], so if the scattered wave is circularly polarized, matched to a left-hand circularly polarized (LHC) antenna, then $s_{4}$ will be +1 (and -1 for a right-hand circular (RHC) antenna match). In some texts there is a minus sign for $s_{4}$ [2], but these implicitly employ the alternative forward scattering alignment (FSA) coordinate system. Note that while $s_{1}$ is always non-negative, $s_{2,3,4}$ are all bipolar variables, depending on the transmitter state $T$ and the nature of the scatterer. Classically there are two main types of Stokes vector; polarized (when $\operatorname{det}(\mathrm{C} 2)=0$ ) and unpolarized (when only $s_{1}$ is non-zero and the signal is noise-like). It is well known [13] that any Stokes vector can be decomposed into the sum of these two components, as shown in (3) where $\operatorname{det}\left(\mathbf{C} 2_{p}\right)=0$.

$$
\mathrm{C} 2=\mathrm{C} 22_{p}+\frac{n}{2}\left[\begin{array}{ll}
1 & 0 \\
0 & 1
\end{array}\right] \rightarrow \underline{s}=\underline{s_{p}}+n \underline{s_{n}}, \underline{s_{n}}=\left[\begin{array}{l}
1 \\
0 \\
0 \\
0
\end{array}\right]
$$

Here, $s_{p}$ and $n \underline{s_{n}}$ represent the polarized and the unpolarized Stokes vectors, respectively. $n$ is the total noise power, and it can be straightforwardly estimated directly for this model from the degree of polarization of the wave $D_{p}$, as shown in (4).

$$
n=s_{1}-\sqrt{s_{2}^{2}+s_{3}^{2}+s_{4}^{2}}=s_{1}\left(1-D_{p}\right)
$$

Such a model could be applied to dual-pol SAR data, where $n$ is associated with the addition of thermal noise in the image. However, it is a key proposal of this paper that this is not the best strategy for dealing with physical interpretation of the Stokes vector in dual-pol SAR applications. Still key to our process, however, will be the idea of extracting a component of $\mathrm{C} 2$ that has zero determinant, $\mathrm{C} 2{ }_{p}$, representing a polarized wave, and so we now turn to consider these states in more detail.

\section{A. Parameterization of polarized waves}

The set of all elliptically polarized waves can be mapped onto the surface of a sphere, the Poincaré sphere, as shown in Fig. 1 [2], [3].

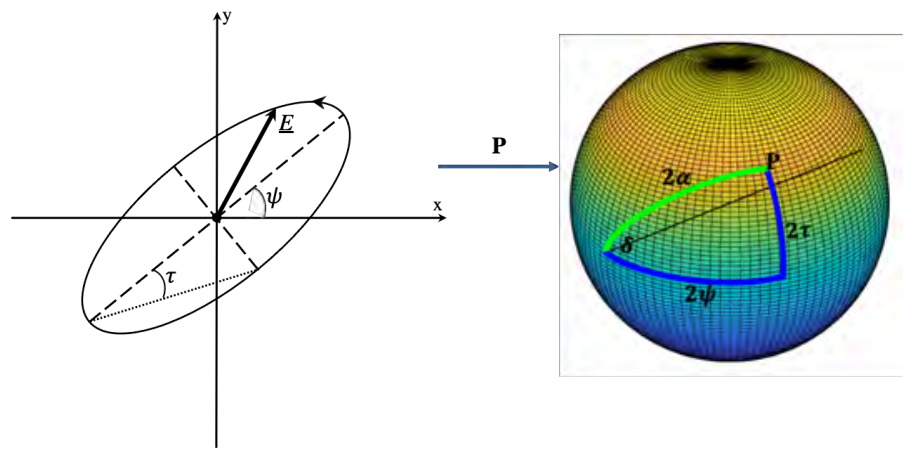

Fig. 1. The Poincaré sphere and spherical triangle construction relating ellipse geometry $(\psi, \tau)$ and wave ratio $(\alpha, \delta)$ parameters for general elliptical polarization state $\mathrm{P}$.

An elliptical polarization state $\mathrm{P}$ can then be characterized by its geometry, the ellipse major axis orientation $\psi$, and ellipticity angle $\tau$, and these locate $\mathrm{P}$ on the sphere as the longitude and latitude, respectively (blue lines in Fig. 1). However, there is a second pair of angles available to locate P, forming a spherical triangle as shown in Fig. 1 (green curve). These two angles $(\alpha, \delta)$ relate to the amplitude and phase of the complex polarization ratio defining the state P. Any polarized Stokes vector can then be written in terms of either 
set of these angles, related as shown in (5), where $A$ is the ellipse amplitude.

$$
\underline{s_{p}}=A^{2}\left[\begin{array}{c}
1 \\
\cos 2 \psi \cos 2 \tau \\
\sin 2 \psi \cos 2 \tau \\
\sin \tau
\end{array}\right] \leftrightarrow A^{2}\left[\begin{array}{c}
1 \\
\cos 2 \alpha \\
\sin 2 \alpha \cos \delta \\
\sin 2 \alpha \sin \delta
\end{array}\right]
$$

Here we employ the ratio parameters ( $\alpha$ and $\delta$ ), as these, we will see, can give some physical interpretation in surface and volume scattering. We note that we can estimate these two angles directly from a polarized Stokes vector as shown in (6).

$$
\left.\begin{array}{l}
\alpha=\frac{1}{2} \cos ^{-1}\left(\frac{s_{2}}{s_{1}}\right) \\
\delta=\arg \left(s_{3}+i s_{4}\right)
\end{array}\right\} \rightarrow \underline{P}=\left[\begin{array}{c}
\cos \alpha \\
\sin \alpha e^{i \delta}
\end{array}\right]
$$

According to the BSA system, we therefore employ the notation in (7) for the phase term $\delta$, locating left-hand circular (LHC) at the north pole of the Poincaré sphere in Fig. 1. Note the change in sign between the measured column vector used to form $\mathrm{C} 2$ and the state vector $\underline{P}$. This is because in BSA the surface of the sphere actually represents antenna states matched to a wave and not the wave itself.

$$
\left\{\begin{array}{l}
R H C \quad \mathrm{C} 2=\left[\begin{array}{l}
1 \\
i
\end{array}\right]\left[\begin{array}{cc}
1 & -i
\end{array}\right] \rightarrow \delta=-\frac{\pi}{2} \rightarrow \underline{P}=\left[\begin{array}{c}
1 \\
-i
\end{array}\right] \\
L H C \quad \mathrm{C} 2=\left[\begin{array}{c}
1 \\
-i
\end{array}\right]\left[\begin{array}{ll}
1 & i
\end{array}\right] \rightarrow \delta=\frac{\pi}{2} \rightarrow \underline{P}=\left[\begin{array}{c}
1 \\
i
\end{array}\right]
\end{array}\right.
$$

The reason for employing BSA is that we can then directly map the Stokes parameters to elements of the (BSA) $3 \times 3$ scattering coherency matrix $\mathrm{T} 3$, as widely used in radar polarimetry [1], [2], [4]. From these we will determine a physical approach to dual-pol decomposition.

\section{B. Stokes vector as a projection of the scattering matrix}

A general T3 matrix has 9 elements, and the full mapping of these into the dual-pol Stokes vector is complicated and offers little physical insight [5]. To proceed, we consider instead a subset of such matrices, those showing reflection symmetry, which can always be factored using a rotation matrix $\mathrm{R}$ as shown in (8), where $\theta$ represents the angle mismatch between the symmetry axis of the scatterer and the $\mathrm{H}$ and $\mathrm{V}$ axes of the radar. These then have 6 free parameters.

$$
\begin{gathered}
\mathrm{T} 3 \mathrm{R}=\mathrm{R}(\theta)\left[\begin{array}{ccc}
t_{11} & t_{12} & 0 \\
t_{12}^{*} & t_{22} & 0 \\
0 & 0 & t_{33}
\end{array}\right] \mathrm{R}(-\theta) \\
\mathrm{R}(\theta)=\left[\begin{array}{ccc}
1 & 0 & 0 \\
0 & \cos 2 \theta & -\sin 2 \theta \\
0 & \sin 2 \theta & \cos 2 \theta
\end{array}\right]
\end{gathered}
$$

These do however encompass most models used in the radar literature for rough surface and volume scattering and hence allow us to make physical based decompositions [4], [5]. The general mapping of reflection symmetry into the dual-pol Stokes vector is shown in (9), with upper sign for $\mathrm{H}$ transmit and lower for V. It is important to underline that this mapping is specifically defined for the dual-pol case, and should not be confused with the compact pol (CP) formulation, which also employs Stokes vectors. Full details of the CP case can be found in [14]. We note that, as long as $\theta$ is non-zero, we can have a full observed Stokes vector from dual-pol systems. This emphasizes the importance of collecting fully coherent dual-pol SLC data.

$$
\underline{s}=\frac{1}{2}\left[\begin{array}{c}
\left(t_{11}+t_{22}+t_{33}\right) \pm 2 \cos 2 \theta \operatorname{Re}\left(t_{12}\right) \\
2 \cos 2 \theta \operatorname{Re}\left(t_{12}\right) \pm\left(t_{11}+\cos 4 \theta\left(t_{22}-t_{33}\right)\right) \\
2 \sin 2 \theta \operatorname{Re}\left(t_{12}\right) \pm \sin 4 \theta\left(t_{22}-t_{33}\right) \\
2 \sin 2 \theta \operatorname{Im}\left(t_{12}\right)
\end{array}\right]
$$

We now consider three important examples of this mapping from coherency matrix to Stokes vector, namely Bragg surface scattering, random volume scattering and Pauli phase shifters. These we will use later to interpret S1 decomposition data.

1) Stokes vector for tilted Bragg surface scattering: Bragg surface scattering has a fully polarimetric rank-1 T3R model as shown in (10). Here $m_{s}$ is the total polarized power, while the scattering alpha angle, $0 \leq \alpha_{s} \leq 45^{\circ}$ (not to be confused with $\alpha$ in(5)), depends on the local angle of incidence. The angle $\theta$ depends on the local azimuth slope of the surface [2]. Note that, although we introduce (10) as a specific scattering model, it can be simply extended to any rank-1 symmetric mechanism (with a diagonal scattering matrix from simple rotation) by adding a phase to the $t_{12}$ element, thus including a much wider class of problems. However, we choose the $t_{12}$ phase as $\pi$ to match the Bragg model for purposes of the decomposition proposed in the paper.

$$
\begin{aligned}
\mathrm{T} 3 \mathrm{R} & =2 m_{s} \mathrm{R}(\theta) \\
& \cdot\left[\begin{array}{ccc}
\cos ^{2} \alpha_{s} & -\cos \alpha_{s} \sin \alpha_{s} & 0 \\
-\cos \alpha_{s} \sin \alpha_{s} & \sin ^{2} \alpha_{s} & 0 \\
0 & 0 & 0
\end{array}\right] \mathrm{R}(-\theta)
\end{aligned}
$$

This model can then be mapped into a dual-pol Stokes vector as shown in (11), with upper sign for $\mathrm{H}$ transmit and lower for V. Here we note that for zero surface slope $\left(\theta=0^{\circ}\right)$, the return is always copolarized with the transmitter, i.e., reflection like a simple mirror. However, for sloped surfaces we obtain extra structure in the $s_{3}$ Stokes parameter. We also note the extreme case of $\alpha_{s}=45^{\circ}$ as a dipole scatterer (complete absorption of one linear polarization).

$$
\underline{s}=m_{s}\left[\begin{array}{c}
1 \mp \cos 2 \theta \sin 2 \alpha_{s} \\
-\cos 2 \theta \sin 2 \alpha_{s} \pm\left(\cos ^{2} \alpha_{s}+\cos 4 \theta \sin ^{2} \alpha_{s}\right) \\
-\sin 2 \theta \sin 2 \alpha_{s} \pm \sin 4 \theta \sin ^{2} \alpha_{s} \\
0
\end{array}\right]
$$

This occurs for very large incidence angle surface scattering or in some crop scattering, such as rice [15], [16], where strongly oriented volume scatter causes differential absorption. The large incidence angle surface example maintains $\mathrm{V}$ polarization (with no $\mathrm{H}$ ) but the vertically oriented crops absorb $\mathrm{V}$ and maintain only $\mathrm{H}$. Such orientation differences can easily be accommodated in the model of (10) by considering surface scatter for $\theta<45^{\circ}$ and vertical crops for $\theta>45^{\circ}$. We can also extend this model to include dihedral scattering (when 
$\alpha_{s}>45^{\circ}$ and $\theta=90^{\circ}$ ). Again, for dihedrals we have orientation effects causing structure in the $s_{2}$ and $s_{3}$ Stokes parameters. Note however that, unlike quad-pol systems, we cannot easily distinguish surface from dihedral scattering from the dual-pol Stokes vector itself.

2) Stokes vector for Random Volume Scattering: Random volume scattering is characterized by the stronger physical constraint of full azimuthal symmetry (3-D space filling of random orientations). In this case $\mathrm{T} 3 \mathrm{R}$ is diagonal, with equal $t_{22}$ and $t_{33}$ coefficients [2], [5]. This model has the form shown in (12), with $m_{v}$ being the volume power. Here, the single particle shape parameter, $F_{p}$, ranges from 0 (scattering from random dihedral particles) to infinity (scattering from spheres), and it is 2 for dipoles. It is important to underline that $F_{p}=1$ corresponds to strong multiple scattering, and not to an ideal depolarizer. Recall that the latter cannot be realized in the backscattering case, due to the reciprocity theorem [5]. In fact, the $4^{\text {th }}$ eigenvalue of the underlying T4 matrix is always 0 , and even setting $F_{p}=1$, the T4 eigenvalues spectrum is $\lambda_{1,2,3,4}=[1,1,1,0]$, which does not generate a randomly polarized wave for all incident states.

$$
\mathrm{T} 3 \mathrm{R}=\frac{2 m_{v}}{F_{p}+2}\left[\begin{array}{ccc}
F_{p} & 0 & 0 \\
0 & 1 & 0 \\
0 & 0 & 1
\end{array}\right] \quad 0 \leq F_{p}<\infty
$$

Therefore (12) maps into the dual-pol Stokes vector as shown in (13).

$$
\underline{s}=\frac{m_{v}}{F_{p}+2}\left[\begin{array}{c}
F_{p}+2 \\
\pm F_{p} \\
0 \\
0
\end{array}\right] \stackrel{F_{p}=2}{\longrightarrow} \underline{s}=m_{v}\left[\begin{array}{c}
1 \\
\pm 0.5 \\
0 \\
0
\end{array}\right]
$$

Note that this volume Stokes vector is only truly randomly polarized when $F_{p}=0$. For all other volume shape factors $F_{p}$, we obtain a partially polarized wave, especially for the important example of a random dipole cloud, $F_{p}=2$, when we obtain a degree of polarization equal to 0.5 , as shown in (13). This random dipole cloud model is widely used in quad-pol decompositions [6], [7] (such as the Freeman-Durden and Yamaguchi algorithms) and hence will be used here to illustrate dual-pol analysis.

3) Stokes vector for a tilted Pauli phase: Finally, we consider how physical structure can be observed even in the $4^{\text {th }}$ Stokes parameter, $s_{4}$. So far, we have seen that for both tilted surface scattering and volume scattering, this parameter remains zero. While it is always possible to consider isolated scattering from helices [7] in the scene to directly generate circular polarization from linear, it is fair to say that such types of scatterer are not generic in remote sensing applications. Here we show, by a simple example, how such structure can in fact be observed in many natural media without the need to invoke helical scatterers. The model we use is non-zero Pauli phase in T3R [14]. A simple form of this model is shown in (14).

$$
\mathrm{T} 3 \mathrm{R}=\mathrm{R}(\theta)\left[\begin{array}{ccc}
1 & e^{i \phi} & 0 \\
e^{-i \phi} & 1 & 0 \\
0 & 0 & 0
\end{array}\right] \mathrm{R}(-\theta)
$$

Here we have a 2-parameter model, a rotated phase shifter, with $\phi$ being the Pauli phase. Note that (14) has zero helicity [2]. The projected dual-pol Stokes vector is then shown in (15).

$$
\underline{s}=\left[\begin{array}{c}
1 \pm \cos 2 \theta \cos \phi \\
\cos 2 \theta \cos \phi \pm \frac{1}{2}(1+\cos 4 \theta) \\
\sin 2 \theta \cos \phi \pm \frac{1}{2} \sin 4 \theta \\
\sin 2 \theta \sin \phi
\end{array}\right]
$$

Here we see that $s_{4}$ can be nonzero under two important physical conditions. Firstly, that the rotation angle $\theta$ is nonzero, and secondly that the Pauli phase is also not zero or $\pi$. The latter has been confirmed in many natural media, ranging from forests to land-ice [1] and is widely satisfied. The former requires specific conditions of topography variation and twisting forces to misalign the scatterer axis with the radar coordinates and as such will only occur in specific applications at specific times. We shall investigate these later in S1 data. These examples illustrate the need to develop a more generalized decomposition approach as an alternative to the standard polarized-plus-noise decomposition shown in (3). We now turn to consider how to formulate a general approach to decomposition to allow for arbitrary partially polarized volume terms in dual-pol data.

\section{Generalized Stokes Vector Decomposition}

The basic ingredients of any model-based decomposition are a model for volume scattering and a means of extracting (uniquely) one or more rank-1 (polarized) remainder terms. As pointed out in the introduction, dual-pol systems are unable to perform such full decomposition, due to their limited information compared to quad-pol. However, we can construct a direct analog for the Stokes vector by combining a volume model with a polarized wave. One problem we face is that dual-pol volume models are not randomly polarized (13), and hence we need to find a unique way to decompose an arbitrary Stokes vector into partially polarized and polarized wave components. We start by modeling the observed Stokes vector as a sum of 3 components, as shown in (16).

$$
\underline{s}=m_{v} \underline{s}_{v}+m_{s} \underline{s}_{p}+n \underline{s}_{n}
$$

Here $n$ is a noise term and $s_{n}$ a randomly polarized Stokes vector as shown in (3). This we assume can either be ignored or mitigated separately by using noise subtraction techniques [17]. Hence, we have a 2-term decomposition to consider. For example, if we adopt a random dipole cloud as our volume model (see (13)), then the decomposition takes the explicit form shown in (17), with upper sign for $\mathrm{H}$ transmit and lower for $\mathrm{V}$.

$$
\underline{s}=m_{v}\left[\begin{array}{c}
1 \\
\pm 0.5 \\
0 \\
0
\end{array}\right]+m_{s}\left[\begin{array}{c}
1 \\
\cos 2 \alpha \\
\sin 2 \alpha \cos \delta \\
\sin 2 \alpha \sin \delta
\end{array}\right]
$$

We see that now we have 4 unknowns in the model and 4 observables in $\underline{s}$. Key to finding a unique solution is to use the fact that $\underline{s}_{p}$ is always polarized. Algebraically, this can be expressed by a metric equation as shown in (18), where $G$ is a 
$4 \times 4$ matrix, as shown, which belongs to a well known family of matrices employed in the Stokes algebra [5].

$$
\mathrm{G}=\left[\begin{array}{cccc}
1 & 0 & 0 & 0 \\
0 & -1 & 0 & 0 \\
0 & 0 & -1 & 0 \\
0 & 0 & 0 & -1
\end{array}\right] \rightarrow\left\{\begin{array}{l}
\operatorname{det}\left(\mathrm{C} 2_{p}\right)=0 \\
\underline{s}_{p}^{T} \mathrm{G} \underline{s}_{p}=0
\end{array}\right.
$$

This implies that the following equation must hold (19), allowing us to find $m_{v}$ by solving a quadratic as shown in (20).

$$
\left(\underline{s}-m_{v} \underline{s}_{v}\right)^{T} \mathrm{G}\left(\underline{s}-m_{v} \underline{s}_{v}\right)=0
$$

The quadratic coefficients $a, b$ and $c$ are shown both in general terms for an arbitrary volume model $s_{v}$ and, on the far right, for the special case of a dipole cloud.

$$
\begin{gathered}
a m_{v}^{2}+b m_{v}+c=0 \\
\downarrow \begin{array}{c}
\downarrow \\
a=\underline{s}_{v}^{T} \mathrm{G} \underline{s}_{v}=0.75 \\
b=-2 \underline{s}^{T} \mathrm{G} \underline{s}_{v}=-2 s_{1} \pm 0.5 s_{2} \\
c=\underline{s}^{T} \mathrm{G} \underline{s}=s_{1}^{2}-s_{2}^{2}-s_{3}^{2}-s_{4}^{2}
\end{array}
\end{gathered}
$$

Only one root of this quadratic will satisfy energy conservation $\left(m_{v} \leq s_{1}\right)$ providing a unique solution for the volume power $m_{v}$. The polarized component can now be found by simple subtraction (21), noting that this will always provide a physical Stokes vector due to selection of the quadratic root to conserve energy. Hence, we avoid all the negative eigenvalue issues that hinder some quad-pol model-based approaches [18].

$$
\underline{s}-\hat{m}_{v} \underline{s}_{v}=\hat{m}_{s} \underline{s}_{p}
$$

From the polarized Stokes vector component, we can then determine the angles $\alpha$ and $\delta$ and construct the state vector $\underline{P}$ as shown in (22). This is then a measure of the polarized contribution relevant to a given pixel and carries information about the nature of the scatterer. Note that in (22) we choose to select the $\alpha$-origin $\left(\alpha=0^{\circ}\right)$ always at the transmitter state $\mathrm{H}$ or $\mathrm{V}$. We can shift this origin simply by using the upper sign for $\mathrm{H}$ transmit and the lower for $\mathrm{V}$ transmit systems. This makes physical interpretation of the $\alpha$ angle simpler. It is then always zero when the return is copolarized with the transmitter (whether $\mathrm{H}$ or $\mathrm{V}$ ), and $90^{\circ}$ when orthogonal. A detailed description of the relationship between $\alpha, \theta$ and $\alpha_{s}$ is provided in the Appendix.

$$
\left.\begin{array}{l}
\alpha=\frac{1}{2} \cos ^{-1}\left(\frac{ \pm s_{2 p}}{s_{1 p}}\right) \\
\delta=\arg \left(s_{3 p}+i s_{4 p}\right)
\end{array}\right\} \rightarrow \hat{m}_{v}, \hat{m}_{s}, \underline{\hat{P}}=\left[\begin{array}{c}
\cos \alpha \\
\sin \alpha e^{i \delta}
\end{array}\right]
$$

We now turn to apply this new decomposition approach to different sample data sets, taken from the C-band S1 SAR satellite system operated by ESA.

\section{DeCOMPosition OF SENTINEL-1 DATA}

There has already been extensive research carried out into exploitation of dual channel polarimetric data from the ESA Sentinels, some including phase information in the full $\mathrm{C} 2$ matrix. However, most of these have been based on either data classification studies [19], [20] (without physical modelling) or employing the model-free entropy-alpha eigenvector decomposition approach [21]. In related work, secondary physical parameters such as radar-vegetation-index (RVI) have been used [22], and while such parameters have an implicit scattering model in mind, the full parameterization of such a model has not yet been realized. In this study we present, we believe for the first time, analysis of S1 data based on a general approach to physical model-based decomposition. Here we apply the generalized Stokes vector decomposition (see Section II-C) to S1 data which cover different scenarios, agricultural, forest, urban and glacial land-ice, thus showing the potential of this TD method when dealing with dual-pol S1 observations.

\section{A. Dataset Description and Pre-Processing}

In this study, we use four different datasets: the first and the second from RS2, acquired over the San Francisco Bay area (USA) and an agricultural area in Sevilla (Spain), respectively. The third dataset, from $\mathrm{S} 1$, is collected over the same agricultural site, and the final one from S1 over glaciers in Svalbard (Norway). These datasets provide us with both time series and angle of incidence diversity but are all collected at the same wavelength (C-band). Analysis at longer wavelengths (especially L-band, where extensive space-borne dual-pol datasets are also available), will be the subject of future studies.

The RS2 imagery consists of full-polarimetric observations, which are here exploited to compare decomposition results obtained as a function of the two main options for dualpol combination, made available by transmitting $\mathrm{H}$ or $\mathrm{V}$, i.e., $\mathrm{HH}-\mathrm{HV}$ and VH-VV. The first dataset is an image collected on April 9, 2008, in fine-quad-pol mode, which corresponds to beam FQ9 (ascending pass). Such an image covers the San Francisco Bay (whose geographical center is $37.5^{\circ} \mathrm{N}$, $122.3^{\circ} \mathrm{W}$ ), a widely studied site for the analysis of TD approaches (e.g., see [2]). In fact, different land cover types are present, i.e., ocean, forest and urban areas which are differently oriented. The technical characteristics of this image are summarized in Table I, while a Google Earth picture of the the San Francisco Bay is shown in Fig. 2(a), where forest areas are highlighted in green, and two urban districts, named "D1" and "D2", are highlighted in red. The second RS2 dataset consists of a time series of images gathered in wide finequad-pol mode in 2014, from May 22 to September 26, which overlaps three different orbits with beams FQ8W, FQ13W and FQ19W (see details listed in Table II). These images cover an agricultural area (with geographical center in $37.1^{\circ} \mathrm{N}, 6.1^{\circ}$ W) with rice as dominant crop type, and other land covers: urban areas, a river, and scattered forested areas. Fig. 2(b) shows a Google Earth image of the whole Sevilla site. Here, the main rice area is highlighted in orange, while the forest (urban) areas are highlighted in green (red). Regarding the area highlighted in cyan, this is the so called "BXII area", where other crops are cultivated [23]. Pre-processing of the RS2 datasets comprises the following steps: radiometric calibration (look-up-table to $\sigma^{0}$ ); formation of the $\mathrm{C} 2$ matrices for each dual-pol combination; speckle filtering $(9 \times 9$ boxcar $)$, and geocoding. In the case of the Sevilla dataset, coregistration is 
also undertaken to ensure that all the images have a pixel-bypixel correspondence.

The first dataset of S1 images was acquired in 2018 over the same geographical area, including 32 images gathered by both satellites, S1A and S1B, providing an effective a 6-day revisit period. These images correspond to the Interferometric Wide Swath (IW) VH-VV polarimetric mode, which is the standard mode over land in Europe. The main features of such images are reported in Table III. Finally, the last dataset is composed of two S1 images acquired on May 24 and May 25, 2021, over the Nordaustlandet island, in the Svalbard archipelago, located at $79^{\circ} \mathrm{N}, 18^{\circ} \mathrm{E}$. This region has been widely studied using airborne radar data [24]. Conveniently for us, over polar regions the $\mathrm{S} 1$ dual-pol combination of channels changes for different passes, and hence we can obtain IW S1 images corresponding to both dual-pol combinations studied, i.e., VHVV and HH-HV. A Google Earth image of the Svalbard site is shown in Fig. 2(c), where the images footprints are also highlighted. These two images cover entirely the Vestfonna ice cap (the smaller glacier), and a large part of the Austfonna ice cap. The details of these images are described in Table IV. The pre-processing steps for all S1 images were implemented using the ESA-SNAP software (https://step.esa.int/main/) as follows: Apply-Orbit-File, TOPSAR-Split, thermal noise removal on the complex backscattering amplitude (using the method we proposed in [17]), radiometric calibration, TOPSAR-Deburst, Slice-Assembly, Subset (to select only an excerpt of the whole image), formation of the C2 matrix, speckle filtering $(4 \times 20$ boxcar), and geocoding. Also in this case, for the Sevilla test site, all the S1 images are stacked in SNAP, to ensure that they share the same geographical grid.

\section{B. Decomposition steps and RGB/HSV representations}

For a given dual-pol image, thermal noise is removed from the full C2 during pre-processing [17], and the Stokes vector of the backscattered wave $\underline{s}$ is formed from the noise-free C2 (2). Thereafter, we decompose $\underline{s}$ according to the 2-layer model in (17), where we have a volume and a polarized term, with the volume modeled as a cloud of random dipoles. Thus, we obtain a unique solution for $m_{v}$ by (18)-(20), and the parameters of the polarized term, $m_{s}, \alpha$ and $\delta$, are found from the remainder (21).

In order to better visualize the outputs of the decomposition for each pixel of the image, we also employ false-color RGB and HSV representations aimed at providing physically motivated color visualization when dealing with S1 dual-pol data.

The RGB composite is used to assess which term of the decomposition, whether the volume or the polarized one, dominates the backscattered polarization state. Hence, the Red (R) and Green $(\mathrm{G})$ channels are $\mathrm{R}=10 \log _{10}\left(m_{s}\right)$ and $\mathrm{G}=$ $10 \log _{10}\left(m_{v}\right)$. Regarding the Blue (B) channel, we define the ratio $r_{s v}$ as $r_{s v}=m_{s} / m_{v}$, and we set $\mathrm{B}=10 \log _{10}\left(r_{s v}\right)=\mathrm{R}$ $-\mathrm{G}$.

Regarding the HSV color-coding, this is employed to represent the information carried by the cross-polarized phase $\delta$. Here, the Hue is equal to $\delta$ (scaled in the range $\pm 180^{\circ}$ ),

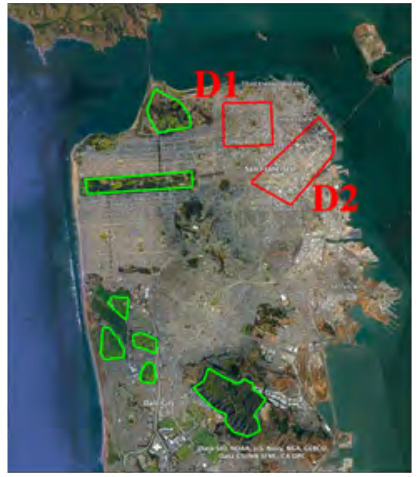

(a)

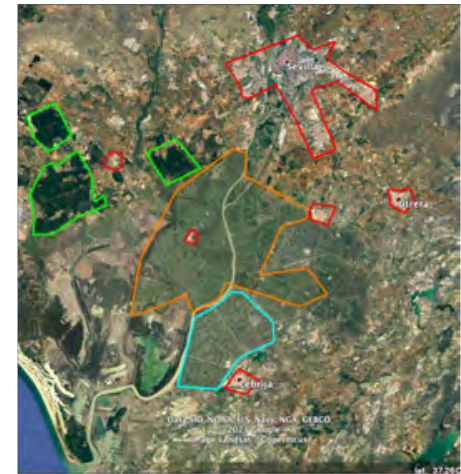

(b)

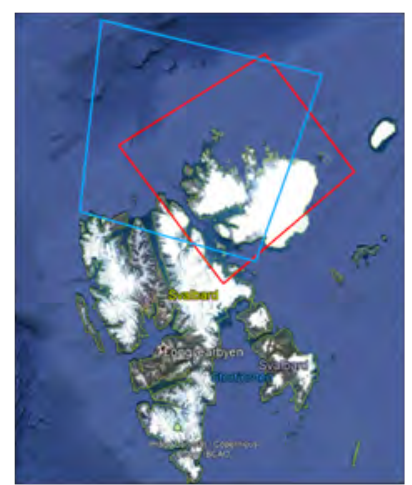

(c)

Fig. 2. (a) Google Earth image of the San Francisco Bay area, where two urban districts (D1 and D2) are highlighted in red, and forest areas highlighted in green. (b) Google Earth image of the Sevilla test site with the green, orange, cyan and red polygons indicating forest, rice, agricultural and urban areas, respectively. (c) Google Earth image of the Svalbard test site. The blue and the red polygons corresponds to the images acquired on May 24 and May 25, 2021, respectively.

while the Saturation component is the coherence between the polarimetric channels, obtained from $\mathrm{C} 2$ as shown in (23).

$$
\rho=\left|\frac{c_{12}}{\sqrt{c_{11} c_{22}}}\right|
$$

Finally, the Value term is the total backscattered power (the span of C2). The HSV color-coding is shown in Fig. 3. According to this scheme, areas in the image characterized by low coherence (noisy $\delta$ ) will be displayed with conventional gray scale modulated by the span, indicating no useful phase information. On the other hand, those areas where phase is important (high coherence) will be colored according to $\delta$ values, with $\pm 180^{\circ}$ corresponding to red, and $0^{\circ}$ to cyan.

\section{Decomposition of dual-pol Radarsat-2 Data}

1) San Francisco dataset: We start by showing the results from the first RS2 dataset relevant to the San Francisco Bay area. In this case, the decomposition is applied on the two dual-pol combinations, $\mathrm{HH}-\mathrm{HV}$ and $\mathrm{VH}-\mathrm{VV}$, resulting in two sets of parameters: $m_{s, H H H}, m_{v, H H V}, \alpha_{H H H V}, \delta_{H H H V}$; $m_{s, V H V V}, m_{v, V H V V}, \alpha_{V H V V}, \delta_{V H V V}$. Fig. 4 shows the RGB, the HSV and the alpha angle images for these data, with the 
TABLE I

RADARSAT-2 DATASET OVER SAN FRANCISCO (USA)

\begin{tabular}{cccccc}
\hline Beam & Date & $\begin{array}{c}\text { Incidence } \\
\text { angle }\left(^{\circ}\right)\end{array}$ & $\begin{array}{c}\text { Azimuth } \\
\text { resolution } \\
(\mathrm{m})\end{array}$ & $\begin{array}{c}\text { Slant-range } \\
\text { resolution } \\
(\mathrm{m})\end{array}$ & $\begin{array}{c}\text { Number } \\
\text { of } \\
\text { images }\end{array}$ \\
\hline FQ9 & $09 / 04 / 2008$ & 28 & 7.6 & 5.2 & 1
\end{tabular}

TABLE II

RADARSAT-2 DATASET OVER SEVILla (SPAIN)

\begin{tabular}{cccccc}
\hline Beam & Dates & $\begin{array}{c}\text { Incidence } \\
\text { angle }\left({ }^{\circ}\right)\end{array}$ & $\begin{array}{c}\text { Azimuth } \\
\text { resolution } \\
(\mathrm{m})\end{array}$ & $\begin{array}{c}\text { Slant-range } \\
\text { resolution } \\
(\mathrm{m})\end{array}$ & $\begin{array}{c}\text { Number } \\
\text { of } \\
\text { images }\end{array}$ \\
\hline FQ8W & $22 / 05 / 2014-19 / 09 / 2014$ & 28 & 7.6 & 5.2 & 6 \\
FQ13W & $22 / 06 / 2014-26 / 09 / 2014$ & 33 & 7.6 & 5.2 & 5 \\
FQ19W & $05 / 06 / 2014-09 / 09 / 2014$ & 39 & 7.6 & 5.2 & 6
\end{tabular}

TABLE III

SENTINEL-1 DATASET OVER SEVILla (SPAIN)

\begin{tabular}{cccccc}
\hline $\begin{array}{c}\text { Relative } \\
\text { orbit }\end{array}$ & Dates & $\begin{array}{c}\text { Incidence } \\
\text { angle }\left(^{\circ}\right)\end{array}$ & $\begin{array}{c}\text { Azimuth } \\
\text { spacing }(\mathrm{m})\end{array}$ & $\begin{array}{c}\text { Slant-range } \\
\text { spacing }(\mathrm{m})\end{array}$ & $\begin{array}{c}\text { Number } \\
\text { of } \\
\text { images }\end{array}$ \\
\hline 74 & $28 / 05 / 2018-30 / 11 / 2018$ & 39 & 13.84 & 2.33 & 32
\end{tabular}

TABLE IV

SENTINEL-1 DATASET OVER SVALBARD (NORWAY)

\begin{tabular}{cccccc}
\hline Relative orbit & Date & $\begin{array}{c}\text { Incidence } \\
\text { angle }\left({ }^{\circ}\right)\end{array}$ & $\begin{array}{c}\text { Azimuth spacing } \\
(\mathrm{m})\end{array}$ & $\begin{array}{c}\text { Slant-range } \\
\text { spacing }(\mathrm{m})\end{array}$ & Polarisation \\
\hline 154 - Descending & $24 / 05 / 2021$ & 39 & 13.84 & 2.33 & VH-VV \\
174 - Ascending & $25 / 05 / 2021$ & 42 & 13.84 & 2.33 & HH-HV
\end{tabular}

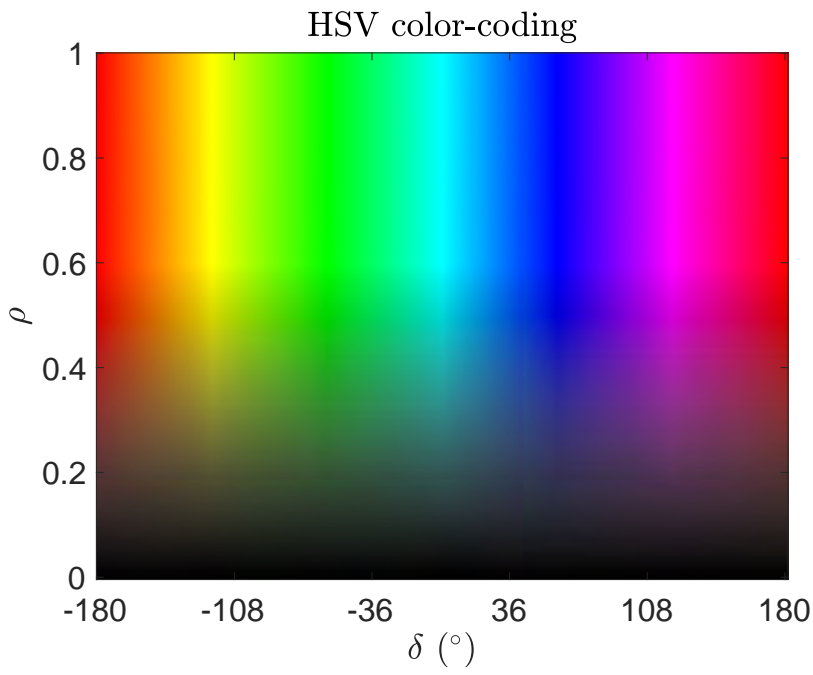

Fig. 3. HSV color-coding employed in this study. Hue: polarimetric phase $(\delta)$, Saturation: cross-polarized coherence $(\rho)$ and Value: span.

upper (bottom) row corresponding to the HH-HV (VH-VV) dual-pol combination. Note that the radar coordinates are also annotated in the figure.

In the case of the ocean, Bragg surface scattering is in place, for which the polarized term dominates the backscattered state, for both the dual-pol modes. Hence, $m_{s}>>m_{v}\left(r_{s v}>>\right.$
1 ), with high (negligible) $m_{s}\left(m_{v}\right)$ values. This leads to a dominance of both the $\mathrm{R}$ and the $\mathrm{B}$ channels (the $\mathrm{G}$ channel has no contribution) in the RGB images (see Figs. 4(a),(d)), for which blue/violet colors are observed. Regarding the HSV images in Figs. 4(b),(e), these are colored in gray, indicating no phase information. This is expected, since the $\mathrm{H}-\mathrm{V}$ axes of the radar are aligned with the symmetry axes of the sea surface (i.e., $\theta \approx 0^{\circ}$ ), for which $\rho$ in (23) is very low. Accordingly, $\alpha$ is also very low (mainly below $\sim 3^{\circ}$ ) for both the HH$\mathrm{HV}$ and the VH-VV mode (see Figs. 4(c),(f)), indicating that polarization state of the polarized term is always copolarized with the transmitted one.

In the case of land, the decomposition outputs clearly vary, depending on the land cover type. Regarding forested areas, the random-dipole volume term is the main contribution to the backscattered state. Hence $m_{v}>m_{s}$, for which $r_{s v}<1$, corresponding to a dominance of the $\mathrm{G}$ channel, and hence to green colors in the RGBs (for both $\mathrm{HH}-\mathrm{HV}$ and the $\mathrm{VH}-$ $\mathrm{VV}$ ). The lower $r_{s v}$ ratio results in low $\rho$ values, and hence grayscale colors in the HSVs, that is noisy $\delta$. Concerning $\alpha$, low-to-moderate (but noisy) values are observed in both the dual-pol modes, with $\alpha_{H H H V}$ and $\alpha_{V H V V}$ ranging mainly between $0^{\circ}$ and $\sim 50^{\circ}$. Regarding urban areas, we clearly note that these parameters vary as a function of the orientation of the buildings with respect to the radar flight direction. Generally, in this case we expect the polarized term to be the dominant contribution to the backscattered state. However, 


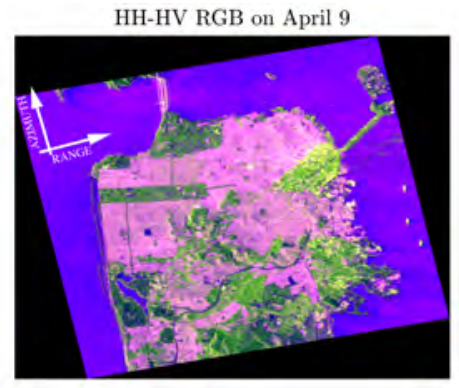

(a)

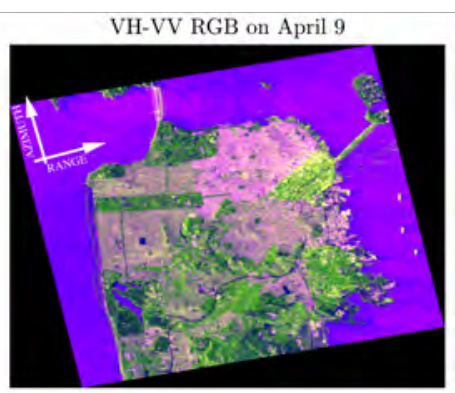

(d)

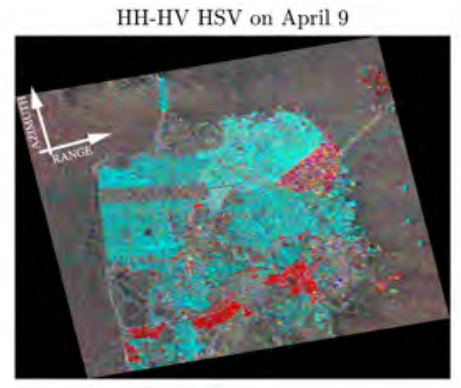

(b)

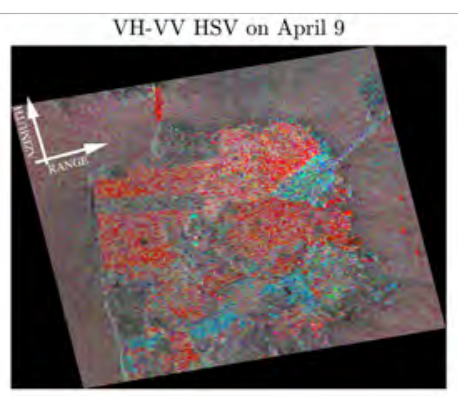

(e)

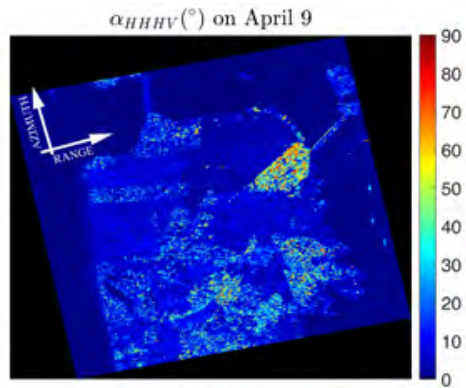

(c)

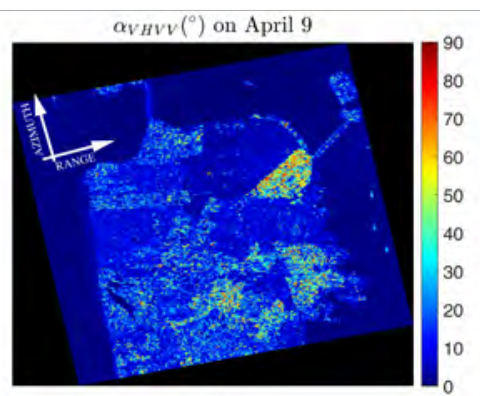

(f)

Fig. 4. Decomposition outputs relevant to the RS2 dataset on the San Francisco Bay area, collected on April 9, 2008, with the upper (bottom) representing the HH-HV (VH-VV) case. (a) and (d) RGB composites with R: $10 \log _{10}\left(m_{s}\right)$ and G: $10 \log _{10}\left(m_{v}\right)$ and B = R - G; (b) and (e) HSV representations; (c) and (f) $\alpha$ angle.

if such areas have a particular orientation, they might result in a higher-than-expected volume term. This is a well known phenomenon which also affects TD approaches for POLSAR data. In this case, the buildings in the D1 district are parallel to the radar flight direction (see also Fig. 2(a)), thus resulting in $m_{s}>>m_{v}\left(r_{s v}>>1\right)$, with high (low, but not negligible) $m_{s}\left(m_{v}\right)$ values. Accordingly, the $\mathbf{R}$ and the $\mathbf{B}$ channels are higher than the $\mathrm{G}$ one in the RGBs, and pink colors are observed for both the dual-pol modes. Note that, also in this case, $\theta$ should be close to $0^{\circ}$. Thus, being $r_{s v}$ high (i.e., high coherence), the HH-HV (VH-VV) is mainly colored in cyan (red) which, according to the HSV color-coding in Fig. 3, corresponds to $\delta_{H H H V} \approx 0^{\circ}\left(\delta_{V H V V} \approx \pm 180^{\circ}\right)$. Note that the shift between $\delta_{H H H V}$ and $\delta_{V H V V}$ is due to the \pm annotation in the Stokes vector formalism, i.e., see $s_{3}$ in (11). Regarding $\alpha$, it is very low, indistinguishable from the sea surface case. Hence, we cannot distinguish among surface and dihedral scattering from the observed polarization state (see discussion in the Appendix). A completely different behavior is observed for district $\mathrm{D} 2$, which consists of strongly oriented buildings. This causes $m_{v}>m_{s}\left(r_{s v}<1\right)$ for the two dual-pol combinations, and thus green colors in the RGBs. Moreover, such orientation misaligns the $\mathrm{H}-\mathrm{V}$ axes of RS2 and the buildings symmetry axes, for which structure in the second and the third elements of $\underline{s}$ in (11) is observed. This results in the opposite HSV colors with respect to the D1 case, where the HH-HV (VH-VV) combination is colored in red (cyan), that is $\delta_{H H H V} \approx \pm 180^{\circ}\left(\delta_{V H V V} \approx 0^{\circ}\right)$. However, these phase values are noisier in this case, as a result of the lower $r_{s v}$ ratio, which indicates a lower cross-polarized coherence. Last but not least, the $\alpha$ angle is significantly larger for both the dual-pol combinations, compared to the D1 case. Here, both $\alpha_{H H H V}$ and $\alpha_{V H V V}$ are mainly larger than $\sim 30^{\circ}$, exhibiting maximum values close to $90^{\circ}$. Therefore, in this case, from Figs. 13(b),(d) in the Appendix, we deduce that $\theta$ should be around $45^{\circ}$, for which such larger $\alpha$ values differ from the sea surface case.

Finally, we also note some orientation effects which are polarization dependent. For instance, this is clearly observed in the two urban areas located on the left side of the images, separated by a forested area. Here, we note pink colors in the HHHV RGB (see Fig. 4(a)), while for VH-VV, the misalignment with the $\mathrm{H}-\mathrm{V}$ radar axes is such that $\mathrm{V}$ - polarized waves are attenuated with respect to the H- polarized ones. Accordingly, $m_{v, V H V V}>m_{s, V H V V}$, for which we observe: greenish RGB colors (see Fig. 4(d)); lower coherence (noisier $\delta_{V H V V}$ ); and moderate $\alpha_{V H V V}$ values (approximately between $30^{\circ}$ and $\left.40^{\circ}\right)$.

2) Sevilla dataset: We now present the results for the RS2 dataset relevant to the Sevilla test site, considering the images collected on June 22 (beam FQ13W, see Table II), August 26 (FQ8W) and September 26 (beam FQ13W), 2014. Figs. 5, 6, and 7 show the RGB, the HSV and the alpha angle for these three images, respectively. In this case, depending on the land cover type, these parameters also vary with time.

Regarding forested areas, they exhibit a stable temporal behavior. Forests always appear in green in the RGBs for both dual-pol combinations and, again, they do not show any useful phase information, with the HSVs being mainly gray. Then, moderate (but noisy) $\alpha$ values are observed for all acquisitions, with $\alpha_{H H H V}$ and $\alpha_{V H V V}$ ranging mainly between $30^{\circ}$ and $50^{\circ}$. Such values suggest that we can reasonably assume 


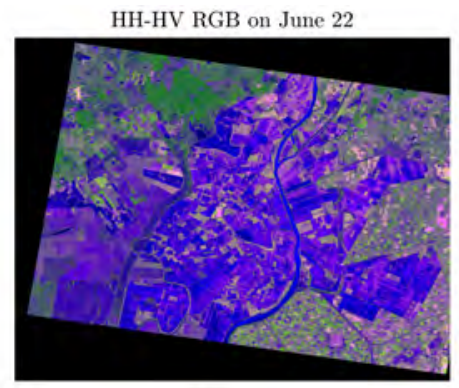

(a)

VH-VV RGB on June 22

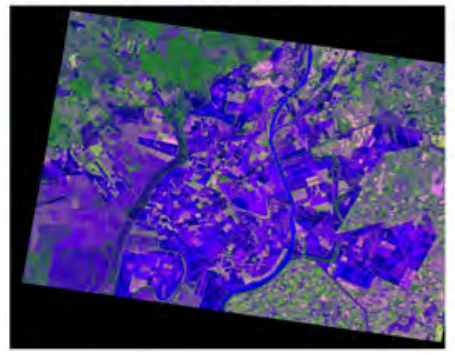

(d)

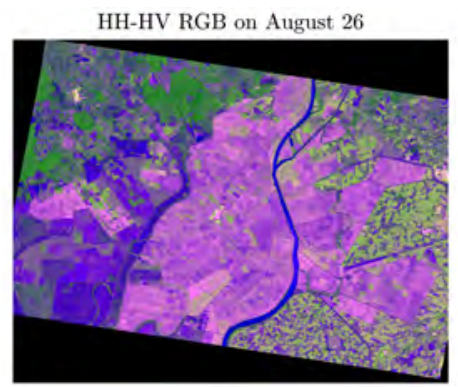

(b)

VH-VV RGB on August 26

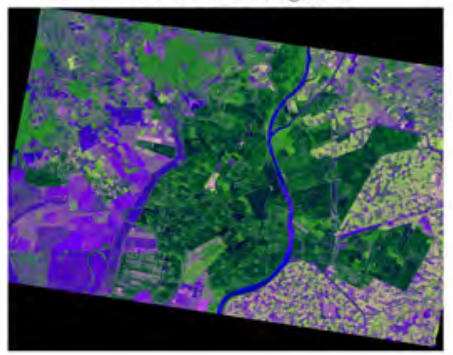

(e)

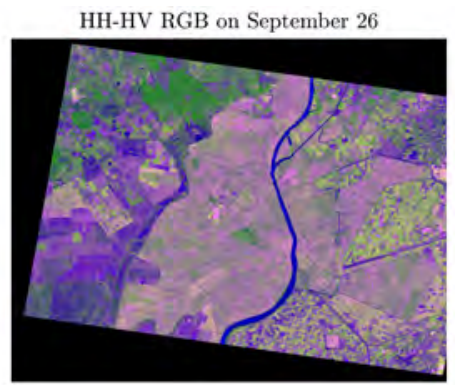

(c)

VH-VV RGB on September 26

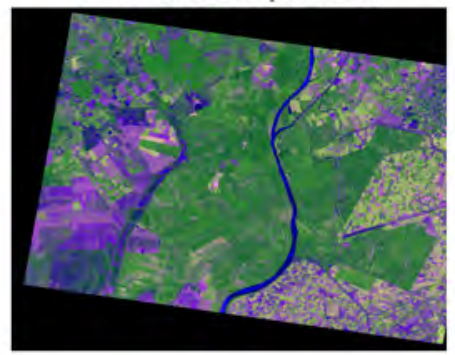

(f)

Fig. 5. RGB composites relevant to the RS2 dataset in Sevilla, for the images collected on June 22, August 26 and September 26,2014 . R $=10 \log _{10}\left(m_{s}\right)$ and $\mathrm{G}=10 \log _{10}\left(m_{v}\right)$ and $\mathrm{B}=\mathrm{R}-\mathrm{G}$. Upper (bottom) row: HH-HV (VH-VV) case.

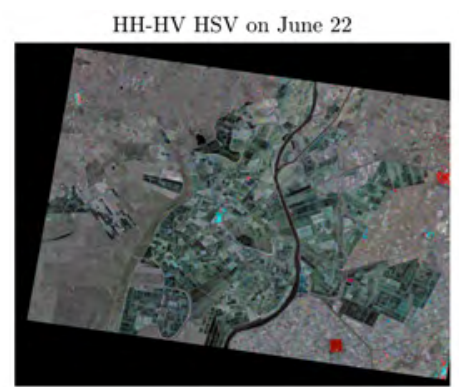

(a)

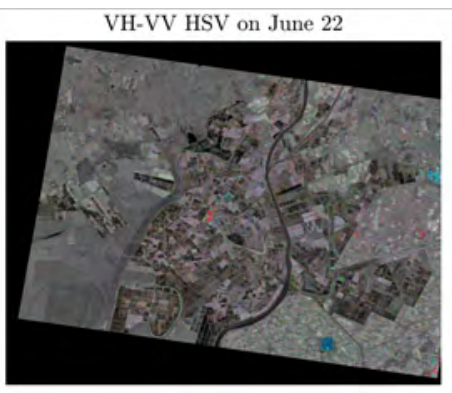

(d)

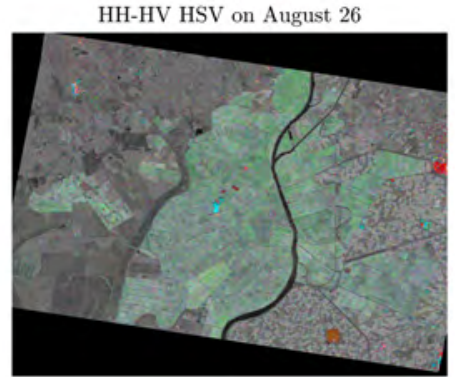

(b)

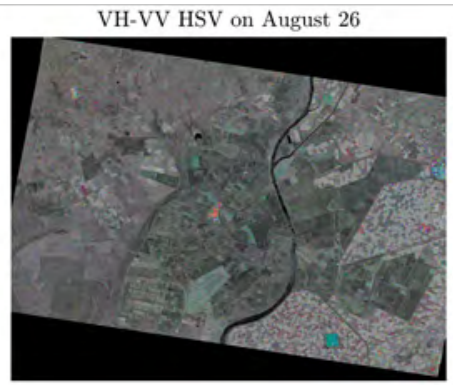

(e)

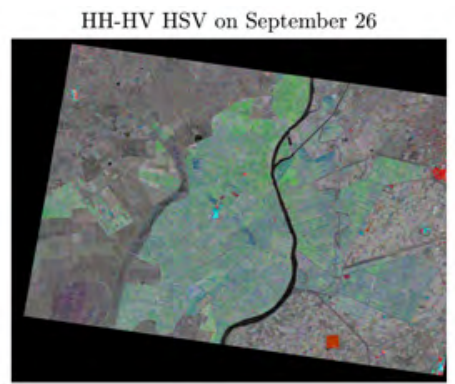

(c)

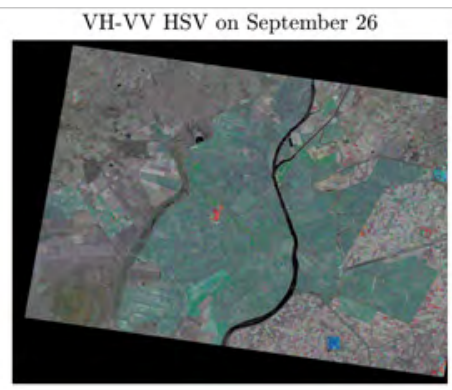

(f)

Fig. 6. HSV representations relevant to the RS2 dataset in Sevilla, for the images collected on June 22, August 26 and September 26, 2014. Upper (bottom) row: $\mathrm{HH}-\mathrm{HV}(\mathrm{VH}-\mathrm{VV})$ case. 


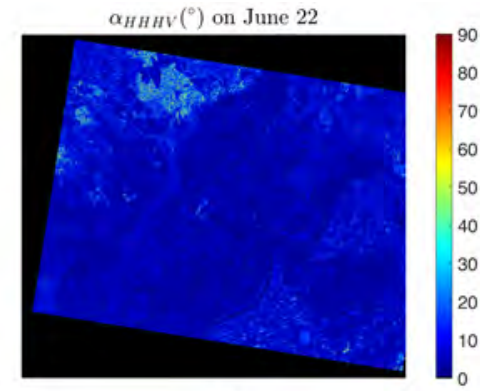

(a)

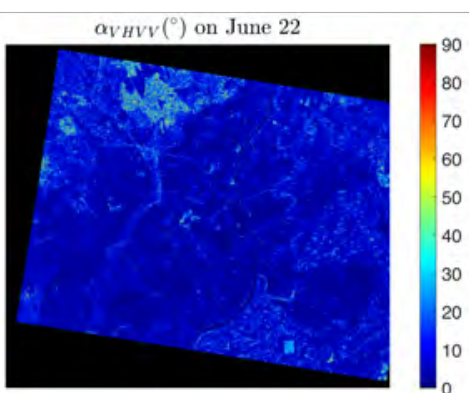

(d)

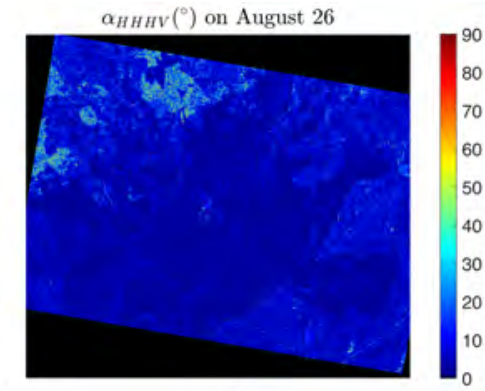

(b)

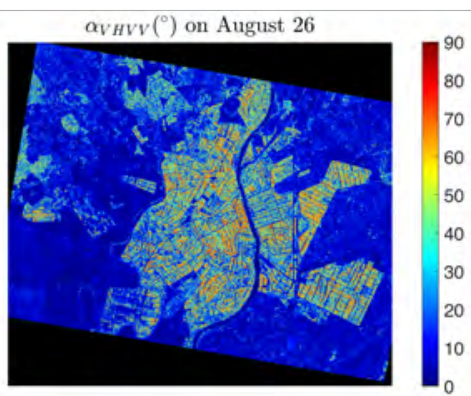

(e)

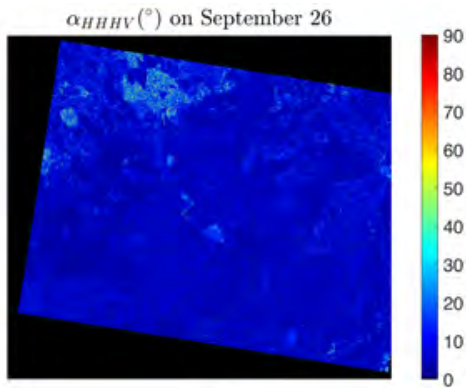

(c)

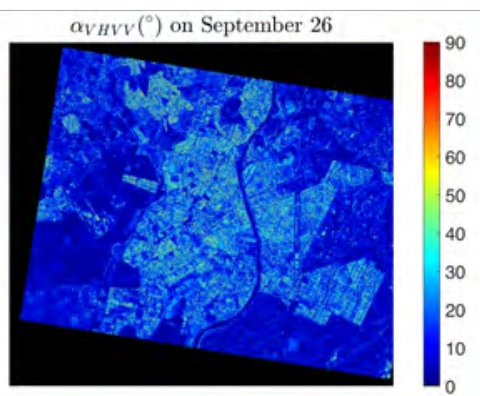

(f)

Fig. 7. Alpha angle relevant to the RS2 dataset in Sevilla, for the images collected on June 22, August 26 and September 26, 2014. Upper (bottom) row: HH-HV (VH-VV) case.

dipole scattering for the polarized term (see Table VII in the Appendix). In particular, we can deduce scattering from weakly oriented dipoles (see the "Almost horizontal" and "Almost vertical" cases in Table VII) which, in the limit, show $\alpha_{H H H V}=\alpha_{V H V V}=45^{\circ}$.

In the case of rice, the parameters of the decompositions exhibit important and more consistent changes as the plants develop in time, with the two dual-pol combinations behaving differently. On the acquisition of June 22, rice plants are at the beginning of the season, for which the polarized contribution from the flooded soil dominates. Accordingly, blue/magenta colors are observed in the two RGBs. Note that the light color variations in the HSV images are driven by some slightly higher $r_{s v}$ values. Regarding alpha, it is low (below $10^{\circ}$ ) for both the HH-HV and the VH-VV mode.

This situation drastically changes on August 26, where significant differences are observed between the two dual-pol modes. These are due to the mainly vertical orientation of the rice plants at this date, for which we can reasonably model the rice stems as "Almost vertical" and "Vertical" dipoles (see Table VII). Accordingly, such vertical stems causes differential extinction [15], for which the V-polarized waves are significantly attenuated with respect to the H-polarized ones. Hence, the behavior of the decomposition outputs is summarized as follows:

- HH-HV case. The polarized term from the underlying soil represents the dominant contribution to the backscattered polarization state. Hence, $m_{s_{H H H V}}>m_{v, H H H V}$, with moderate-to-high (low) $m_{s_{H H H V}}\left(m_{v, H H V}\right)$ values, which results in pink RGB colors (see Fig. 5(b)). Being $r_{s v}>1$, this indicates higher values of the crosspolarized coherence, for which the HSV image, shown in
Fig. 6(b), results in cyan/green colors, i.e., $\delta_{H H H V}$ varies approximately in the range $-100^{\circ}-0^{\circ}$ (see Fig. 3). These phase values are related to the dielectric constant of the plants which, in turn, depend on the water content of the stems. $\alpha_{H H H V}$ is low (see Fig. 7(b)), being mainly below $\sim 20^{\circ}$, as expected for vertical dipoles (Table VII). This denotes that the polarized state is copolarized with the transmitted one.

- VH-VV case. The polarized term from the soil is largely attenuated due to the extinction caused by the rice stems. Accordingly, the volume term is the main contribution to the backscattered state, i.e., $m_{v,,_{V H V}}>>m_{s, V H V V}$ and, as a result, the RGB image (see Fig. 5(e)) is colored in green/dark green. Then, since $r_{s v}<<1$, low coherence values are experienced, thus leading to gray tones in the HSV image (see Fig. 6(e)). As expected, $\alpha_{V H V V}>45^{\circ}$ (see Fig. 7(e)), being mostly around $70^{\circ}$, indicating that the polarized state of the received wave is almost orthogonal to the transmitted one.

For the last image, on September 26, the dominant volume term in the VH-VV case (light green RGB, as shown in Fig. 5(f)) is due to the randomly oriented plant elements in the upper part of the rice canopy at this date, such as panicles, leaves, etc., and it also affects the HH-HV mode, where some greenish areas are observed (see Fig. 5(c)). As for the alpha angle, it decreases up to values around $40^{\circ}$ for VH-VV (see Fig. 7(f)), as a result of the gradual vanishing of the aforementioned extinction phenomenon, while it is still low in the HH-HV case (see Fig. 7(c)). It is interesting to note colorful HSV images for both the dual pol modes at this date (see Fig. 6(c),(f)). The HSV is mainly green with some cyan/bluish shades (cyan/bluish) for the HH-HV (VH- 
VV) case, for which $\delta_{H H H V}\left(\delta_{V H V V}\right)$ is mainly around $-50^{\circ}$ with some values $\sim 50^{\circ}$ (is mainly around $0^{\circ}$ ). Although, for the VH-VV case, $r_{s v}<1$ due to the dominance of the volume term, this ratio is higher compared to the previous acquisition, which leads to less noisy $\delta_{V H V V}$ values. The presence of phase information in this case is likely explained by the several rain events occurred in the four days before the acquisition, resulting in a total amount of water of about $550 \mathrm{~mm}$. This led to a significant increase in the plants water content, which, through changes in the dielectric constant, also affects both $\delta_{H H H V}$ and $\delta_{V H V V}$ (which, unlike urban areas, are no longer separated by a $180^{\circ}$ shift).

The RS2 frame covers in part the agricultural BXII area. In this case, the decomposition outputs for the two dual-pol combinations are, overall, similar at all the dates. In particular, we note that: the volume term is the dominant contribution (mainly green colors in the RGBs); the HSV colors are mainly in gray; alpha is mainly low (it reaches some higher values, around $30^{\circ}$, on June 22 ).

Finally, regarding urban areas, only four small towns (see the four small red polygons, including the Utrera town, in Fig. 2(a)), are covered by the RS2 frame. In this case, results are similar to the ones for the San Francisco Bay area. The main RGB color, stable over time, is pink for the HH-HV and VH-VV combinations, demonstrating a complex but stable scattering environment. Regarding the HSVs, these are colored in cyan and red for the HH-HV and the VH-VV mode, respectively. In the case of $\alpha$, it is mainly around $30^{\circ}$ in both cases, indicating some slight orientation effect.

\section{Decomposition of Sentinel-1 Data}

1) Sevilla dataset: We now analyze the results obtained for the S1 dataset collected over the same area near Sevilla in 2018, in VH-VV mode. Also in this case, we consider only three images of the time series, acquired on June 9, August 26 and October 19. The RGB, HSV and the alpha images are shown in Fig. 8. Again, we note important changes in decomposition parameters with time. The area covered by S1 is also much larger than the RS2 one and includes now the city of Sevilla and its surroundings (see Fig. 2(b)), and the entire BXII area. The results in are in agreement with the ones relevant to the VH-VV combination of the RS2 dataset.

Forest areas are always in green in the RGBs, as a result of the dominant volume term, showing no significant phase information (gray HSVs). $\alpha_{V H V V}$ is noisy, with values around $30^{\circ}-50^{\circ}$, denoting scattering from weekly oriented dipoles.

Regarding rice, the polarized term dominates the backscattered wave on June 9 (RGB in blue/violet, see Fig. 8 (a)), while, in August 26 and October 19, the main contribution is the one from the volume layer (RGBs are green, see Fig. 8(b)(c)), as a consequence of differential extinction. The HSV image is mainly gray while, regarding $\alpha$, it is very low in the first image (see Fig. $8(\mathrm{~g})$ ), then it is high, close to $90^{\circ}$, on August 26 (Fig. 8(h)), and finally decreases to values around $40^{\circ}$ on October 19 (see Fig. 8(i)).

In the case of the BXII area, we note some temporal variation in the RGB colors, likely due to the crops development. On June 9, many fields are colored in green and many others in pink/violet, while these crops mainly appear in greenish/yellow on August 26. Finally, we observe again green and pink/violet colors on October 19. Regarding the HSV and $\alpha_{V H V V}$ images, the former is mainly gray at all the dates, while the latter is stable around $25^{\circ}-30^{\circ}$.

It is interesting to note how the dominant term in the RGB, relevant to the areas surrounding the main rice site and BXII, drastically changes in the last image (see Fig. 8(c)). These are also agricultural areas and, at the first two images, the volume term is, in many cases, the dominant contribution. Then, on October 19 we observe a significant dominance of the polarized state, with the RGB being pink/magenta. This is explained by the fact that, in this period of the year, these crops are usually harvested. Moreover, two significant rain events occurred on October 15 and October 18, resulting in 14 and $11 \mathrm{~mm}$ of water, respectively. Hence, the strong surface scattering contribution from the wet soil results in $m_{s}>>m_{v}$.

Regarding urban areas, the higher backscattered levels result mainly in green RGB colors, due to orientation effects. As expected, phase information is significant in this case, mainly in red and cyan HSV colors, corresponding to $\pm 180^{\circ}$, and $0^{\circ}$, respectively. Regarding $\alpha_{V H V V}$, it is around $20^{\circ}$. Finally, note that we observe high $\alpha_{V H V V}$ values (around $80^{\circ}-90^{\circ}$ ) also in some areas of the river and some other water bodies, in the image of October 19. These are caused by low backscattering areas (very low backscattered intensities), and they must not be confused with the extinction phenomenon in the rice case.

2) Svalbard dataset: We turn to analyze a different potential application, land-ice monitoring using two S1 images collected over the Svalbard glaciers in May 2021, in VH-VV and HH-HV combinations. The outputs of the decomposition are shown in Fig.9, where the upper and the bottom row correspond to the VH-VV and HH-HV acquisition, respectively. The radar coordinates are also shown in the plots. Note that, unlike the Sevilla dataset, the two images are not coregistered and hence they do not share the same geographical grid.

Regarding the sea surface, as expected, we observe: blue/violet RGB colors (see Fig.9(a),(d)), due to the dominance of the polarized term; gray tones in the HSVs; low values of the alpha angle. Then, in those areas between the sea and the bottom part of glaciers (which consist of freeice land [25]), the volume term is the dominant contribution (green RGBs). Accordingly, we still observe gray HSV colors (Fig.9(b),(e)), due to the lower coherence values. Regarding $\alpha$ (Fig.9(c),(f)), it exhibits some noisy values between $\sim 20^{\circ}$ and $\sim 30^{\circ}$ for both the two images.

In the case of the glaciers, the Austfonna (Vestfonna) ice cap reaches an elevation of $\sim 791 \mathrm{~m}(\sim 620 \mathrm{~m})$ above the sea level (a.s.1.) [25], which results in significant azimuth slopes along the perimeter of the caps, i.e., on those areas of transition between the land and the top of the caps. Accordingly, the behavior of the decomposition outputs on these land-ice interfaces (LII) is different from the one observed on the top of the caps. This is especially true for the RGB and the HSV representations, in both the VH-VV and the HH-HV image. As we do not dispose of any ground data, we assume that the surface/subsurface structure of the ice changes with elevation as described in [24], [26]. Lower altitudes ("Ablation zone") 


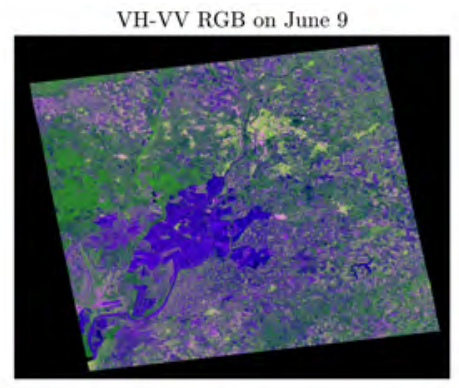

(a)

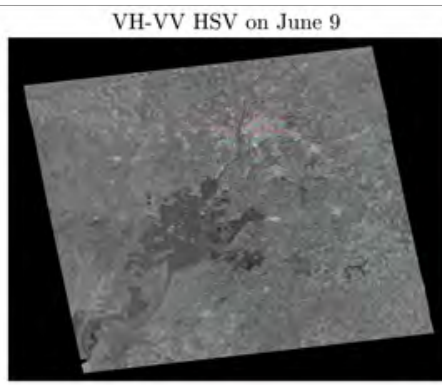

(d)

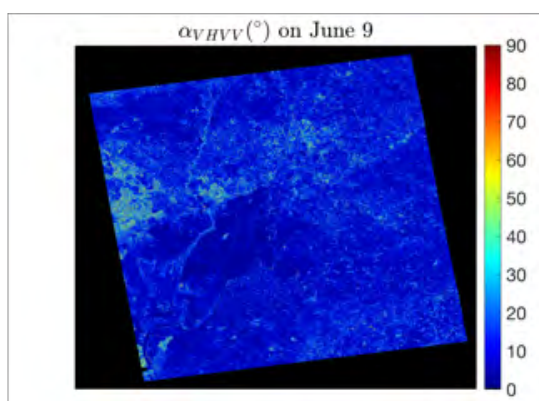

(g)

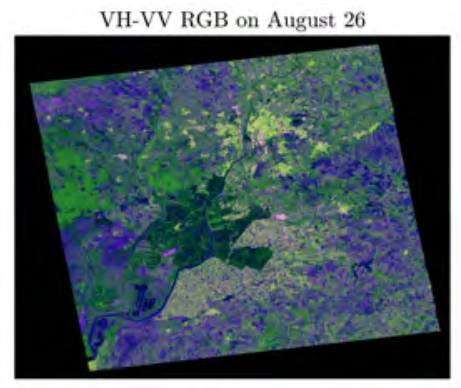

(b)

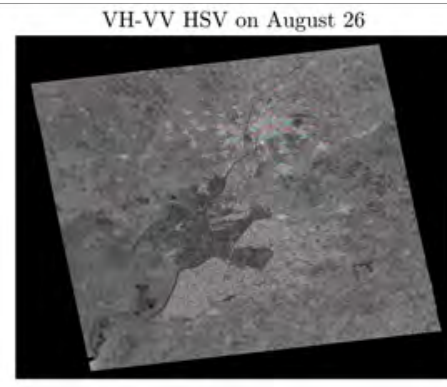

(e)

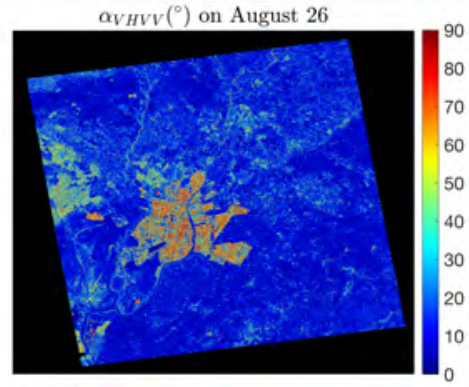

(h)

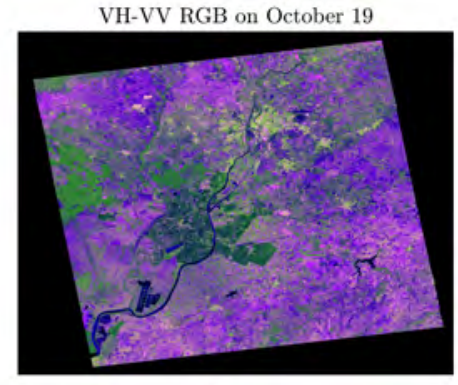

(c)

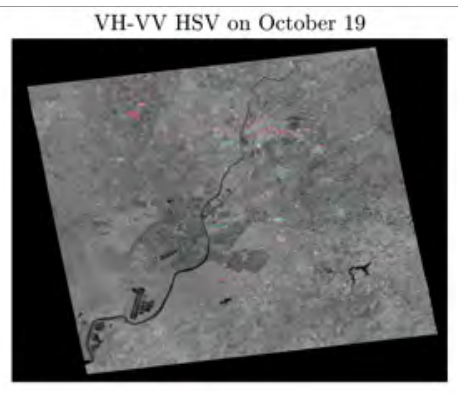

(f)

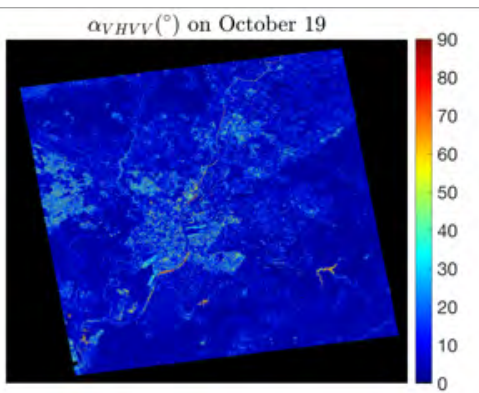

(i)

Fig. 8. Decomposition outputs relevant to the S1 dataset in Sevilla, collected on June 9, August 26, and October 19 2018. (a)-(c) RGB composites; (d)-(f) HSV representations; (g)-(i) $\alpha$ angle.

are characterized by the ice surface lying on top of solid glacier ice. At higher altitudes, oriented layers and randomly distributed inclusions, such as air bubbles and lenses/glands, appear in the ice structure ("Accumulation zone", see [24], [26] for details).

Regarding the LIIs, the polarized term is the dominant contribution. At lower altitudes, this is due to surface scattering at the ice surface, resulting in $m_{s}>>m_{v}$, i.e., pink/magenta RGB colors. Then, as elevation increases, an increase in $m_{p}$ and $m_{v}$ is observed. The former is due to the presence of the oriented ice layers, while the latter is driven by the increasing amount of randomly distributed ice inclusions. Hence, $m_{s}$ is still larger than $m_{v}$, and pink/light orange RGBs are observed. Importantly, since LIIs have high azimuth slopes, the symmetry axes of these scatterers misaligned with the $\mathrm{H}-\mathrm{V}$ radar axes. Consequently, this results in high values of the cross-polarized coherence, for which a clear and spatially extensive phase information is present for both the VH-VV and $\mathrm{HH}-\mathrm{HV}$ image, as shown in the HSVs. We consider this a key result, showing the potential of using the S1 crosspolarized phase for LII monitoring. Other literature studies limit this application to polarimetric radars that provide direct measurement of the copolar-phase-difference (CPD) [24], [26]. Regarding $\alpha$, we observe some relatively higher values in some areas close on the caps perimeter, indicating a transformation of the polarized state of the backscattered wave.

Regarding the top of the caps, a significant dominance of the volume term (i.e., $m_{v}>>m_{s}$ ) is observed. This originates from the large amount of randomly oriented inclusions present in the anisotropic firn layer [24], [26], for which the volume contribution is significantly larger than the polarized one (attributed to the firn). This is in agreement with the results shown in [26] relevant to C-band POLSAR observations. Accordingly, in this case green/yellowish RGBs are observed for the two images, and the low coherence values result in no useful phase information (noisy colors mixed with gray tones shades in the HSVs). Finally, the two images exhibit noticeable differences in the $\alpha$ values on the top of the Austfonna glacier. In fact, in this area, while $\alpha_{V H V V}$ is mainly below $\sim 20^{\circ}$ with some peaks at $30^{\circ}-35^{\circ}, \alpha_{H H H V}$ mostly ranges between $\sim 50^{\circ}$ and $\sim 70^{\circ}$. This is likely caused by the different acquisition geometries, for which such an area is located in 
the near-range (toward the far-range) in the $\mathrm{VH}-\mathrm{VV}$ (HHHV) image. Accordingly, the high $\alpha_{H H H V}$ values are due to surface scattering at higher incidence angle which occurs at the firn/ice (or snow/ice) interface on the top of the cap. This significantly attenuates $\mathrm{H}$-polarized waves with respect to the V-polarized ones (see Appendix).

\section{DISCUSSION AND INTERPRETATION OF RESULTS FOR SENTINEL-1}

In this section, an in depth analysis of the results obtained for the S1 datasets is provided.

\section{A. Results for the Sevilla dataset}

For the Sevilla test site, the results presented in the previous section clearly show important changes of the decomposition outputs with land-cover type, and their variation with time. To better analyze this, we consider four regions of interest (ROIs) corresponding to four different land-covers: an area of the river, a rice field, a forest and an urban area.

Fig. 10(a) shows Google Earth pictures where the polygons corresponding to these ROIs are highlighted. Then, taking benefit of the dense S1 time series (32 images, see Table III), we evaluate the outputs of the decomposition, $m_{v}, m_{s}$ and $\alpha$ (for simplicity, we drop the "VHVV" subscript) for each ROI and for all the acquisitions. In this case, the results in Section III-D1 show that no information is carried by the cross-polarized phase $\delta$ over vegetated (forests and crops) and river areas. Therefore, this parameter is not considered in this analysis.

For each land-cover class, the average values of the decomposition outputs are plotted, against the Day of the Year (DoY), in Fig. 10(b)-(d). The clear differences between these landcover types confirm the results shown in the previous section. In the case of the river, the decomposition outputs depend on the state of the water's surface, which is driven by wind conditions, river's currents, etc. In most of the cases, $m_{s}$ is mainly larger than $m_{v}$, witnessing that the polarized component is the main contribution to the received polarization state. Regarding $\alpha$, it is very low, mainly below $\sim 7^{\circ}$, indicating that the polarized Stokes vector, $\underline{s}_{p}$, is copolarized with the transmitted state. In fact, in this case, Bragg or tilted Bragg surface scattering is in place, characterized, in terms of T3R, by very low values of $\theta$ and $\alpha_{s}$ (see (10) and (11) in Section II-B1). Note that the high alpha value at the last image (around $60^{\circ}$ ), also mentioned in the previous section (see Fig.8(i)), is due to some complex scattering, usually experienced when analyzing water bodies, which departures from the Bragg/tilted Bragg surface scattering. This results in very low backscattered VV intensity, which causes high $\alpha$ values. In the case of rice, $m_{v}$, $m_{s}$ and $\alpha$ change according to its phenological stages. At the beginning of the season, between DoY 156 (sowing date) and DoY $184, m_{s}$ is larger than $m_{v}$, i.e., the polarized term dominates backscattered polarization state, as a consequence of the Bragg/tilted Bragg scattering from the water layer which underlies the emerging rice plants. Accordingly, $\alpha$ is low, mainly below $10^{\circ}$. Then, from DoY 190 up to the end of the season (the field is harvested on DoY 319), we note a clear dominance of the volume term, with $m_{v}$ being always larger than $m_{s}$, due to the increasing amount of plant elements. In particular, $m_{v}$ increases to around $-13 \mathrm{~dB}$ at DoY 220, driven by the developing tillers, which are randomly oriented. Here $m_{s}$ is approximately stable at $\sim-19 \mathrm{~dB}$. Then, up to DoY 238, both $m_{v}$ and $m_{s}$ are attenuated of about $4 \mathrm{~dB}$. This is due to the vertical orientation of the rice stems (stem elongation/booting stages) which imposes a larger attenuation on the V-polarized waves due to extinction [15], thus resulting in lower $m_{v}$ and $m_{s}$ values. The influence of the vertical rice stems in the polarized term is effectively seen in the $\alpha$ angle, which reaches its maximum at DoY 238 (around $70^{\circ}$ ). This indicates that, as a consequence of the vertical dipole scattering (see Table VII in the Appendix), the polarized term is not V-polarized, but almost H-polarized. Finally, from DoY 238 onward, the attenuation due to extinction vanishes, since the upper part of the canopy develops (panicle, heads, etc.) and the vertical stems start drying. Accordingly, $\alpha$ decreases until $\sim 10^{\circ}$. Regarding the forest, the partially polarized state from the volume layer always dominates the radar response, as expected, with $m_{v}\left(m_{s}\right)$ being stable in time around -10 $(-17) \mathrm{dB}$. In the case of $\alpha$, it is stable around $35^{\circ}$, indicating somehow a change of the polarization in the polarized term (scattering from weekly oriented dipoles). As for the urban area, according to the green RGB colors observed in Fig. 8, orientation effects leads to similar values of the polarized and volume powers, with $m_{v}$ being $1 \mathrm{~dB}$ larger than $m_{s}$. Accordingly, the alpha angle is stable around $23^{\circ}$.

We now compare $m_{s}$ and $m_{v}$ with the backscattered power in the VV and VH channels, $\left\langle\left|E_{V V}\right|^{2}\right\rangle$ and $\left\langle\left|E_{V H}\right|^{2}\right\rangle$, whose average values are shown in Fig. 10(e),(f), respectively. Regarding $m_{s}$ (see Fig. 10(b)) we note an average separation of $\sim 10 \mathrm{~dB}$ between the forest and the urban class, while this separation is around 4-5 $\mathrm{dB}$ for both $\left\langle\left|E_{V V}\right|^{2}\right\rangle$ and $\left\langle\left|E_{V H}\right|^{2}\right\rangle$. Concerning $m_{v}$ (see Fig. 10(c)), we clearly note that it is almost a scaled replica of $\left\langle\left|E_{V H}\right|^{2}\right\rangle$, for all the landcover types. This comparison would immediately lead to the conclusion that the volume term is the only contribution to the backscattered VH power, which is the implicit assumption commonly made in the literature. In this regard, while depolarization, of course, highly affects $\left\langle\left|E_{V H}\right|^{2}\right\rangle$, it does not represent the only contribution. In fact, by using (5), we can express the backscattered powers as the sum of the polarized and the volume contribution, denoted with subscript $p$ and $v$, respectively, as shown in (24).

$$
\begin{gathered}
\left\langle\left|E_{V V}\right|^{2}\right\rangle=0.75 m_{v}+0.5 m_{s}(1+\cos 2 \alpha) \\
=\left\langle\left|E_{V V}\right|^{2}\right\rangle_{v}+\left\langle\left|E_{V V}\right|^{2}\right\rangle_{p} \\
\left\langle\left|E_{V H}\right|^{2}\right\rangle=0.25 m_{v}+0.5 m_{s}(1-\cos 2 \alpha) \\
=\left\langle\left|E_{V H}\right|^{2}\right\rangle_{v}+\left\langle\left|E_{V H}\right|^{2}\right\rangle_{p}
\end{gathered}
$$

While $\left\langle\left|E_{V V}\right|^{2}\right\rangle_{v}$ and $\left\langle\left|E_{V H}\right|^{2}\right\rangle_{v}$ are scaled replicas of $m_{v}$, interest is focused on the dependence of $\left\langle\left|E_{V V}\right|^{2}\right\rangle_{p}$ and $\left\langle\left|E_{V H}\right|^{2}\right\rangle_{p}$ on $\alpha$. When $\alpha$ is high, $\left\langle\left|E_{V V}\right|^{2}\right\rangle_{p}\left(\left\langle\left|E_{V H}\right|^{2}\right\rangle_{p}\right)$ is low (high), e.g., like in the case of the vertical rice stems which attenuate V- polarizations. On the other hand, $\left\langle\left|E_{V V}\right|^{2}\right\rangle_{p}\left(\left\langle\left|E_{V H}\right|^{2}\right\rangle_{p}\right)$ is expected to be high (low) when $\alpha$ is low, such as on water bodies, when Bragg surface scattering 


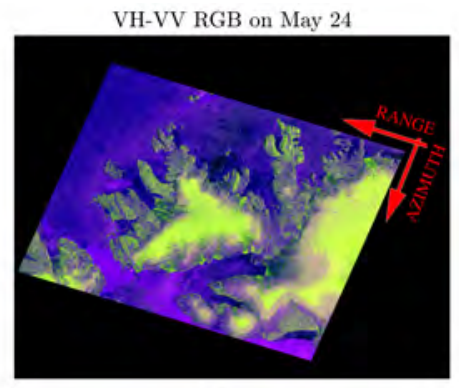

(a)

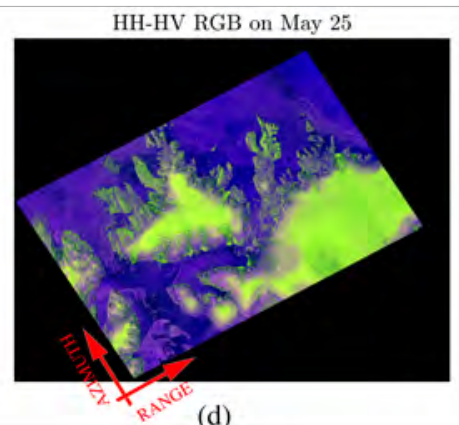

(d)

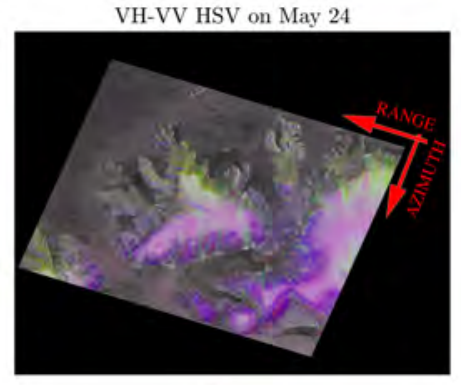

(b)

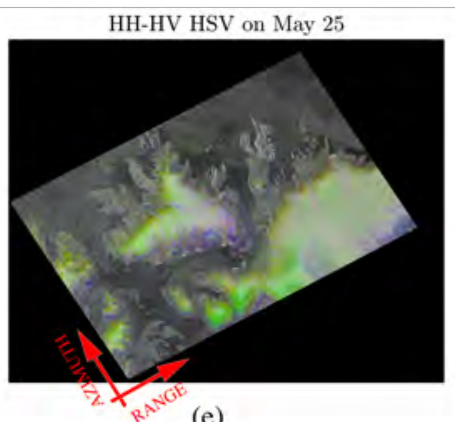

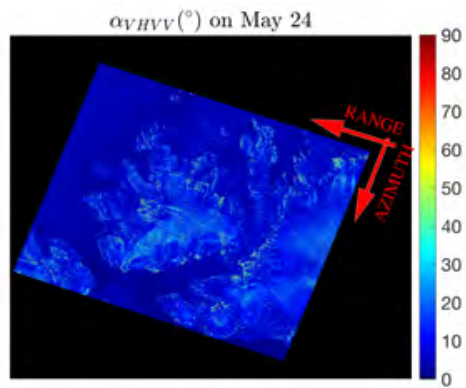

(c)

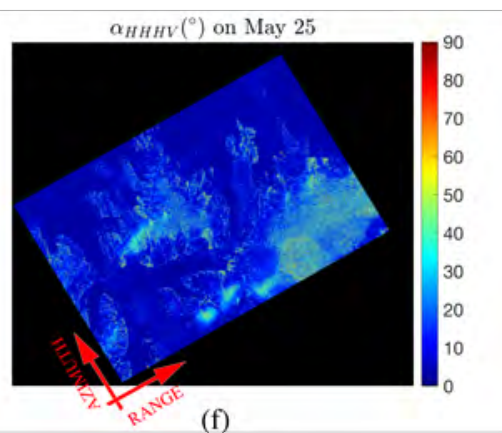

Fig. 9. Decomposition outputs relevant to the S1 dataset collected over Svalbard, in May 24 and May 25. (a)-(c) RGB composite, HSV representation and alpha angle for the VH-VV image, respectively; (d)-(f) RGB composite, HSV representation and alpha angle for the HH-HV image, respectively.

predicts higher (lower) intensity at $\mathrm{V}$ - $(\mathrm{H}-)$ polarization. This dependence on $\alpha$ is then modulated by $m_{s}$.

The average values of $\left\langle\left|E_{V H}\right|^{2}\right\rangle_{p},\left\langle\left|E_{V H}\right|^{2}\right\rangle_{v},\left\langle\left|E_{V V}\right|^{2}\right\rangle_{p}$, $\left\langle\left|E_{V V}\right|^{2}\right\rangle_{v}$ are shown in Fig. 10(g)-(j). The volume contributions are the same as $m_{v}$, only shifted by the proper factor (see (24)). Regarding the river, $\left\langle\left|E_{V H}\right|^{2}\right\rangle_{p}\left(\left\langle\left|E_{V V}\right|^{2}\right\rangle_{p}\right)$ is very low (high) being around $-43 \mathrm{~dB}$ (ranging between -23 and $-13 \mathrm{~dB}$ ), as expected for Bragg surface scattering. Here, $\left\langle\left|E_{V H}\right|^{2}\right\rangle_{v}$ is the main contribution to the $\mathrm{VH}$ power. In the case of the rice field, the volume contribution to the crosspolarized power is always larger than the polarized one. However, the latter exhibits a significant increase as plant develops. At the beginning of the season (DoYs 156-184), $\left\langle\left|E_{V H}\right|^{2}\right\rangle_{v}$ dominates, being significantly larger than $\left\langle\left|E_{V H}\right|^{2}\right\rangle_{p}$ (which is about $-43 \mathrm{~dB}$ ). Then, while $\left\langle\left|E_{V H}\right|^{2}\right\rangle_{v}$ increases of about $6 \mathrm{~dB}$ up to DoY 220, the increment in $\left\langle\left|E_{V H}\right|^{2}\right\rangle_{p}$ is $\sim 12 \mathrm{~dB}$. Successively, as the vertical rice stems develop, $\left\langle\left|E_{V H}\right|^{2}\right\rangle_{v}$ is attenuated (just like $m_{v}$ ), while the polarized contribution continues increasing (of about $5 \mathrm{~dB}$ ) up to DoY 238, when $\alpha$ is maximum. On this date, $\left\langle\left|E_{V H}\right|^{2}\right\rangle_{p}$ and $\left\langle\left|E_{V H}\right|^{2}\right\rangle_{v}$ are almost the same (being -24.3 and $-23.5 \mathrm{~dB}$, respectively), indicating almost equal contributions from these two terms to the $\mathrm{VH}$ power. Then, while $\left\langle\left|E_{V H}\right|^{2}\right\rangle_{p}$ remains stable around $-27 \mathrm{~dB}$ and finally decreases afterward, $\left\langle\left|E_{V H}\right|^{2}\right\rangle_{v}$ increases, becoming again the dominant contribution. Regarding the contributions to the $\mathrm{VV}$ power, $\left\langle\left|E_{V V}\right|^{2}\right\rangle_{p}$ is mainly larger than $\left\langle\left|E_{V V}\right|^{2}\right\rangle_{v}$ at the beginning of the season, and then the latter dominates for the remaining part of the cultivation cycle. Here, contrarily to $\left\langle\left|E_{V H}\right|^{2}\right\rangle_{p}$, at DoY 238 we observe the minimum $\left\langle\left|E_{V V}\right|^{2}\right\rangle_{p}$ (around $-33 \mathrm{~dB}$ ). For the forest and the urban areas, the volume contribution to $\left\langle\left|E_{V H}\right|^{2}\right\rangle$ is always larger than the polarized one, with $\left\langle\left|E_{V H}\right|^{2}\right\rangle_{v}\left(\left\langle\left|E_{V H}\right|^{2}\right\rangle_{p}\right)$ being $\sim-16$ and $\sim-12 \mathrm{~dB}(-23$ and $-19 \mathrm{~dB})$ for the forest and the urban ROIs, respectively. On the other hand, regarding the VV case, while the urban ROIs exhibits similar values of $\left\langle\left|E_{V V}\right|^{2}\right\rangle_{p}$ and $\left\langle\left|E_{V V}\right|^{2}\right\rangle_{v}$ ( -9 and $-8 \mathrm{~dB}$, respectively), for the forest area the volume contribution is $\sim 10 \mathrm{~dB}$ larger than the polarized one, as a consequence of the larger $\alpha$ values (around $\sim 38^{\circ}$, see Fig. 10(d)) and $m_{v}>m_{s}$ (see Fig. 10(b)(c)).

These results show the importance of the polarized contribution to the $\mathrm{VH}$ and $\mathrm{VV}$ backscattered powers, and its variations with the land-cover types. In fact, $\left\langle\left|E_{V H}\right|^{2}\right\rangle_{p}$ results in an average separation of $\sim 20$ (24) dB between river and forest (between river and the urban) areas. This separation is $\sim 10$ (15) $\mathrm{dB}$ for the $\mathrm{VH}$ power, and $\sim 6 \mathrm{~dB}$ for the VV power. Moreover, while for $\left\langle\left|E_{V V}\right|^{2}\right\rangle_{p}$ the forest/urban separation is, on the average, $\sim 11 \mathrm{~dB}$, the $\left\langle\left|E_{V H}\right|^{2}\right\rangle_{p}$ and $\left\langle\left|E_{V V}\right|^{2}\right\rangle_{p}$ exhibit a significant dynamic range (on the average, 20 and $18 \mathrm{~dB}$, respectively), which is much larger than the $\mathrm{VH}$ and VV ones. This opens the potential of using $\left\langle\left|E_{V H}\right|^{2}\right\rangle_{p}$ and $\left\langle\left|E_{V V}\right|^{2}\right\rangle_{p}$ for the estimation of important crop variables, such as biomass and phenology.

\section{B. Results for the Svalbard dataset}

Regarding the Svalbard test site, as shown in the previous section, the significant topography variations present at the LIIs of the glaciers lead to consistent phase information (colorful HSVs), for both the VH-VV and the HH-HV image. In this section, we analyze this behavior in more detail, focusing our attention on the Vestfonna ice cap (VIC).

An excerpt of the VH-VV and HH-HV HSV images relevant to the VIC, is shown in Fig. 11(a) and (b), respectively, where 


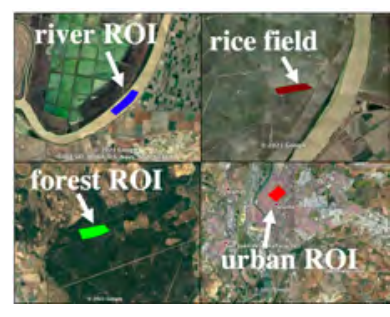

(a)

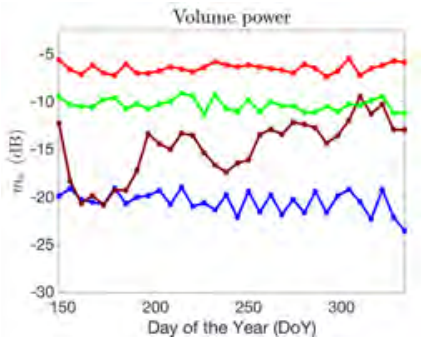

(c)

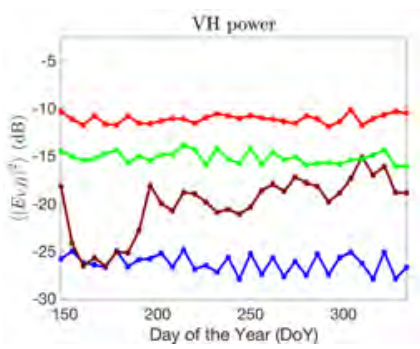

(e)

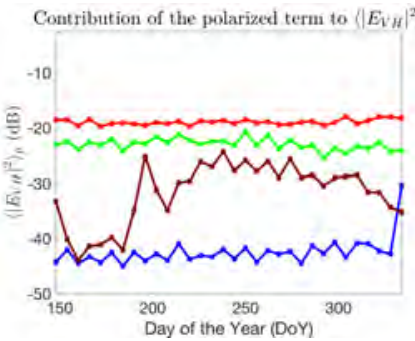

(g)

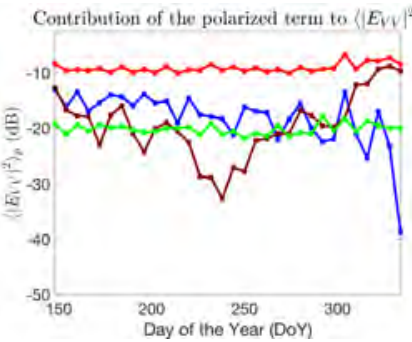

(i)

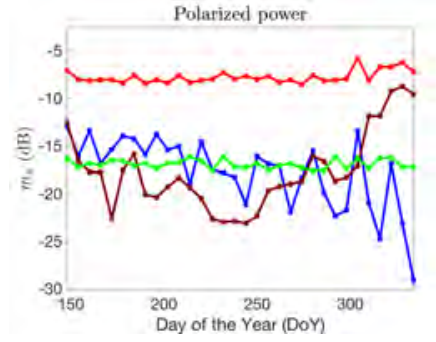

(b)

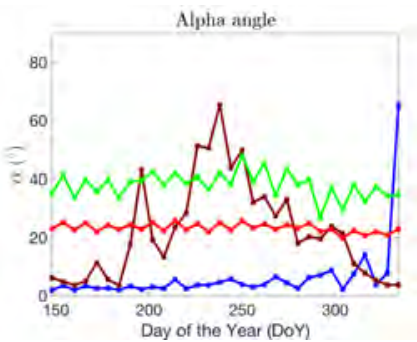

(d)

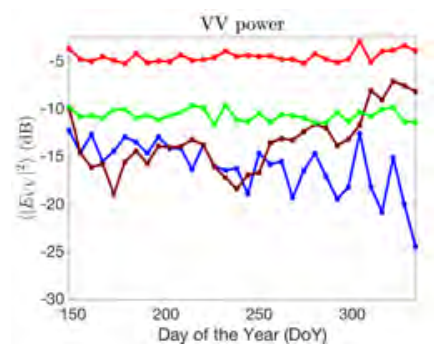

(f)

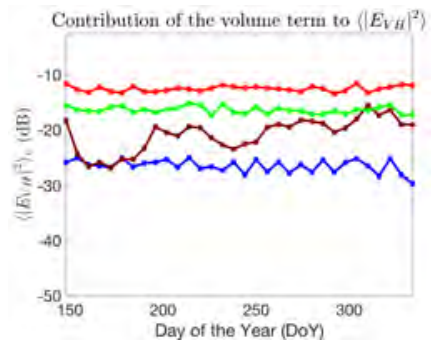

(h)

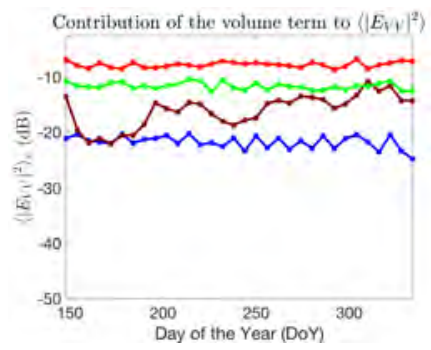

(j)
Fig. 10. Time series of the decomposition outputs for the land-cover classes in the Sevilla test site. (a), Google earth pictures of the land-cover ROIs. (b)-(d) Time series of $m_{s}, m_{v}$ and $\alpha$. (e)-(f) Time series of the VH and VV intensity. (g)-(j) Time series of $\left\langle\left|E_{V H}\right|^{2}\right\rangle_{p},\left\langle\left|E_{V H}\right|^{2}\right\rangle_{v},\left\langle\left|E_{V V}\right|^{2}\right\rangle_{p}$, $\left\langle\left|E_{V V}\right|^{2}\right\rangle_{v}$.

the left (right) column of the figure shows the results for the VH-VV (HH-HV) image.

An important characteristic of the VIC (which reaches $\sim$ $620 \mathrm{~m}$ altitude a.s.1.) is the presence of several outlet glaciers, which drain ice from the cap's center into the surrounding sea [27]. These are clearly distinguished in the HSV maps. In fact, Fig. 11(c) and (d) show an enlargement of the area in the dashed line box in Fig. 11(a),(b), respectively. Here, two outlet glaciers are present (see the black rectangles), characterized by HSV phase patterns, whose colors flow from the top of the cap to the sea.

To further analyze the phase information, we consider its dependence on the azimuth slopes. For such a purpose, we ignore the dependence of $\delta$ on the dielectric constant of the ice. The latter, in terms of the T3R/Stokes mapping (Section II-B), is "hidden" in the Pauli phase (15). Then, we consider, according to the radar pass, upslopes (downslopes) those slopes pointing toward (away from) the radar. Generally, we expect, for both the dual-pol modes, the cross-polarized phase on upslopes to have an opposite sign with respect to the one on downslopes. This can be easily seen in (15), by ignoring the Pauli phase in the $s_{4}$ element. Following this rationale, for each $\mathrm{S} 1$ image, we select two ROIs on two opposite LIIs, named ROI ups and $\mathrm{ROI}_{d w n s}$, corresponding to an upslope and to a downslope, respectively. Note, that since we do not have access to any ground information on the elevation of the cap, we use the data presented in [25] as a reference to roughly identify the altitudes of these ROIs. The ROIs are located in two different areas, which are highlighted in Fig. 11(a)-(b) with a blue and a red box. Then, Figs. 11(e)-(f) (Figs. 11(g)-(h)) show enlargements of these boxes, where $\mathrm{ROI}_{\text {ups }}\left(\mathrm{ROI}_{d w n s}\right)$ are delineated. Note that the ROIs selected for the VH-VV image are different from the ones in the $\mathrm{HH}-\mathrm{HV}$ one, as these images have opposite passes. According to [25], for the $\mathrm{VH}-\mathrm{VV}$ image, $\mathrm{ROI}_{\text {ups }}$ $\left(\mathrm{ROI}_{d w n s}\right)$ has an elevation between 400 and $500 \mathrm{~m}(\sim 350$ $\mathrm{m})$ while, in the $\mathrm{HH}-\mathrm{HV}$ case $\mathrm{ROI}_{\text {ups }}\left(\mathrm{ROI}_{\text {dwns }}\right)$ is located at an altitude of about $200-300 \mathrm{~m}(\sim 500 \mathrm{~m})$. Successively, we evaluate the empirical probability density function (pdf) of the cross-polarized phases, $\delta_{V H V V}$ and $\delta_{H H H V}$, over these ROIs. The latter are shown in Figs. 11(i)-(j). These pdfs have a Gaussian-like shape, with the phase corresponding to $\mathrm{ROI}_{u p s}$ and $\mathrm{ROI}_{\text {dwns }}$ having opposite sign, as expected.

We now show for these two ROIs, the polarization state backscattered from the polarized layer. We consider, for both the VH-VV and HH-HV image, the average values of $m_{s}, \delta$ and $\alpha$, evaluated over the two ROIs, to obtain the amplitudes of the field components, the ellipse orientation angle $\psi$, and the ellipticity angle $\tau$. These average values, along with the average of $m_{s}$ and $m_{v}$, are listed, for the VH-VV and the $\mathrm{HH}-\mathrm{HV}$ image, in Table $\mathrm{V}$ and Table VI, respectively. Note that $m_{v}$ is not involved in the computation of the ellipse parameters, but it is here reported to indicate which of the two terms, either the volume or the polarized one, dominates, on the average, the backscattered wave. The received polarization state from the polarized term is illustrated, for the two ROIs, in Fig. 12, where the first (second) row refers to the $\mathrm{VH}$ VV (HH- HV) case. Note that, in these plots, the direction of propagation points out of the page. As we note, such a polarization state is always an elliptical polarization. This can be interpreted considering the ice structure at higher altitudes (see previous section). In this case, the polarized layer consists of the ice surface and the oriented ice inclusions which appear as elevation increases [24], [26]. As a consequence, the slopes 


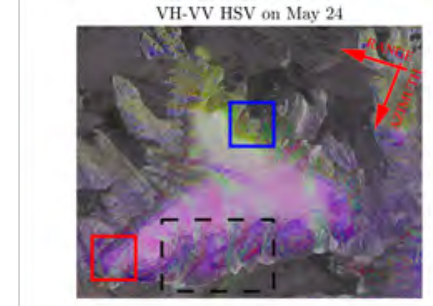

(a)

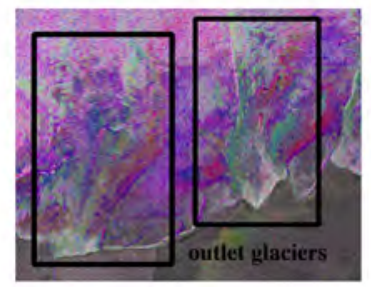

(c)

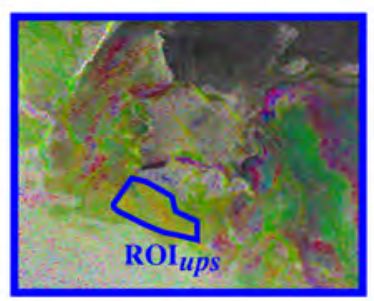

(e)

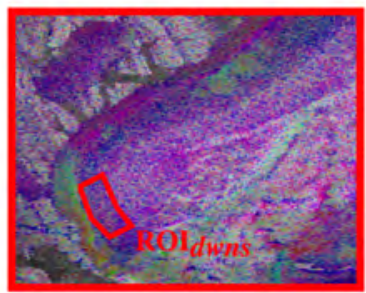

(g)

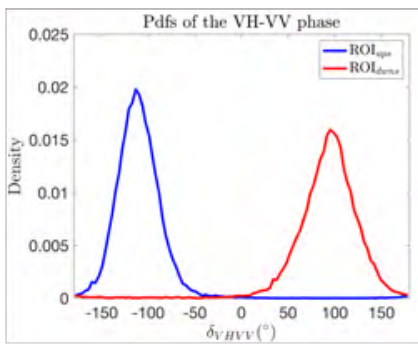

(i)

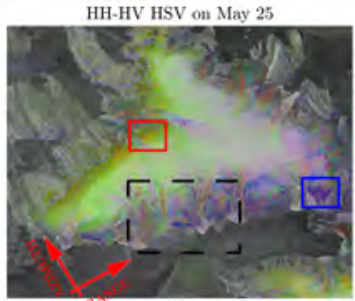

(b)

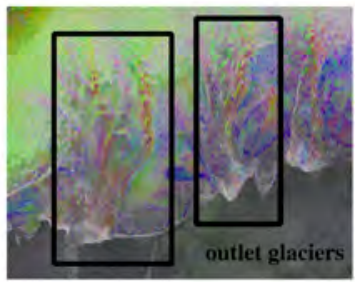

(d)

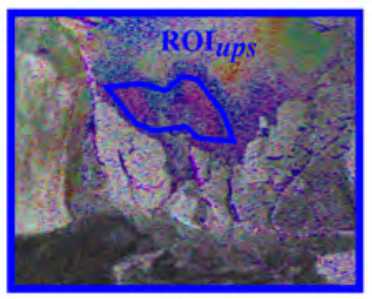

(f)

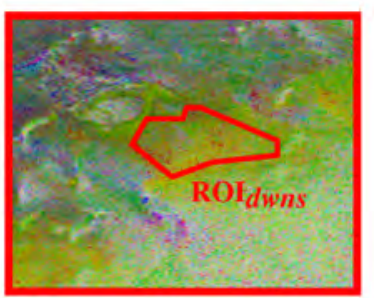

(h)

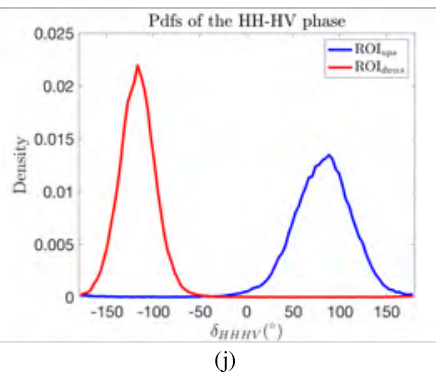

(j)
Fig. 11. Phase analysis on the Vestfonna ice cap. Left column: VH-VV image; right column: HH-HV image. (a)-(b) Excerpts of the HSV images relevant to Vestfonna. The black dashed box highlights outlet glaciers, while the blue and the red boxes highlight different areas (corresponding to opposite slopes). (c),(d) Enlargements of the black dashed box in (a), (b), respectively, where the outlet glaciers are delineated. (e)-(h) Enlargements of the blue and red boxes in (a)-(b), where $\mathrm{ROI}_{u p s}$ and $\mathrm{ROI}_{\text {dwns }}$ are highlighted. (i)-(j) empirical pdfs of the cross-polarized phase evaluated over $\mathrm{ROI}_{u p s}$ and $\mathrm{ROI}_{\text {dwns }}$.

at the LIIs misalign the symmetry axes of such oriented layers and the $\mathrm{H}-\mathrm{V}$ radar axes (i.e., $\theta \neq 0$, see Section II-B), thus leading to $\delta$ values which are neither $0^{\circ}$ nor $180^{\circ}$. This is a key result which shows how this term drastically changes the polarization. Note, also, that no assumption is made on the type of scatterers, i.e., helical (see Section II-B3).

TABLE V

AVERAGE VALUES OF THE ELLIPSE PARAMETERS, ALONG WITH $m_{s}$ AND $m_{v}$, RELEVANT TO ROI $\mathrm{R}_{\text {up }}$ AND ROI R $_{w}$ FOR THE VH-VV IMAGE

\begin{tabular}{ccccccc}
\hline Region & $m_{s}(\mathrm{~dB})$ & $m_{v}(\mathrm{~dB})$ & $\delta\left(^{\circ}\right)$ & $\alpha\left(^{\circ}\right)$ & $\psi\left(^{\circ}\right)$ & $\tau\left(^{\circ}\right)$ \\
\hline $\mathrm{ROI}_{\text {ups }}$ & -8.59 & -9.56 & -109.37 & 14.65 & -5.27 & -13.75 \\
$\mathrm{ROI}_{\text {dwns }}$ & -9.46 & -11.27 & 91.64 & 9.77 & -0.3 & 9.77 \\
\hline
\end{tabular}

TABLE VI

AVERAGE VAlues of THE ELLIPSE PARAMETERS, ALONG WITH $m_{s}$ AND $m_{v}$, RELEVANT TO ROI ups $_{\text {AND ROI }}$ dwns FOR THE HH-HV IMAGE

\begin{tabular}{ccccccc}
\hline Region & $m_{s}(\mathrm{~dB})$ & $m_{v}(\mathrm{~dB})$ & $\delta\left(^{\circ}\right)$ & $\alpha\left(^{\circ}\right)$ & $\psi\left(^{\circ}\right)$ & $\tau\left(^{\circ}\right)$ \\
\hline $\mathrm{ROI}_{\text {ups }}$ & -11.41 & -13.72 & 79.95 & 10.88 & 1.99 & -13.72 \\
$\mathrm{ROI}_{\text {dwns }}$ & -10.21 & -10.36 & -116 & 19.72 & -9.92 & -17.4 \\
\hline
\end{tabular}

Regarding the VH-VV image, in the case of $\mathrm{ROI}_{\text {ups }}$ (located at $\sim 400-500 \mathrm{~m}), m_{p}$ is approximately $1 \mathrm{~dB}$ larger than $m_{v}$ (see Table V). This indicates a lightly larger contribution from the oriented ice inclusions with respect to the randomly distributed ones. $\delta$ and $\alpha$ are around $-109.4^{\circ}$ and $14.7^{\circ}$, respectively, resulting in $\psi \approx-5.3^{\circ}$, and $\tau$ being $\sim-13.8^{\circ}$. Concerning $\mathrm{ROI}_{d w n s}$, the polarized term dominates, with $m_{p}$ being larger than $m_{v}$ of about $2 \mathrm{~dB}$. A possible explanation for this is that, being this ROI at a lower altitude $(\sim 350$ $\mathrm{m})$, the presence of random inclusions in the ice volume is less significant than $\mathrm{ROI}_{\text {ups }}$, thus resulting lower $m_{v}$ values. Here, $\delta(\alpha)$ is $\sim 91.7^{\circ}\left(\sim 9.8^{\circ}\right)$, resulting in $\psi$ and $\tau$ being $\sim-0.3^{\circ}$ and $\sim 9.8^{\circ}$, respectively. These parameters result in a right-handed (left-handed) ellipse for $\mathrm{ROI}_{\text {ups }}\left(\mathrm{ROI}_{\text {dwns }}\right)$, as shown in Fig. 12(a) (Fig. 12(b)). Finally, in the case of the HH-HV image, $m_{p}$ is $\sim 2.3 \mathrm{~dB}$ larger than $m_{v}$ for ROI $\mathrm{R}_{\text {ups }}$ (see Table VI), as this area is located at lower altitudes (200$300 \mathrm{~m}$ ). Accordingly, the contribution from the (randomly oriented) ice lenses/pipes in the ice volume is much less important. Regarding $\delta(\alpha)$, it is $\sim 80^{\circ}\left(\sim 11^{\circ}\right)$, while $\psi$ and $\tau$ are $\sim 2^{\circ}$ and $\sim 10.8^{\circ}$, respectively. A completely different situation is observed for $\mathrm{ROI}_{\text {dwns }}$, whose elevation is much higher $(\sim 500 \mathrm{~m})$. Here, the volume and the polarized term almost equally contribute to the backscattered state, with $m_{p} \approx m_{v} \approx-10.2 \mathrm{~dB}$. This is due to the significant presence of such random inclusions embedded in the firn layer at this high altitudes. Concerning $\delta, \alpha, \psi$ and $\tau$, they are around $-116^{\circ}, 20^{\circ},-10^{\circ}$ and $-17^{\circ}$, respectively. The polarization ellipses (see Figs. 12(c)-(d)) are left- and right-handed, for $\mathrm{ROI}_{\text {ups }}$ and $\mathrm{ROI}_{\text {dwns }}$, respectively.

\section{CONCLUSIONS}

We have advanced a new family of model-based decompositions adapted for dual-pol radar data. The two basic elements of the decomposition are a flexible volume scattering term and a polarized remainder. Here we have employed the widely used random dipole cloud as a volume model, but future studies could look at non-dipole options for a better match to physical scattering mechanisms. 


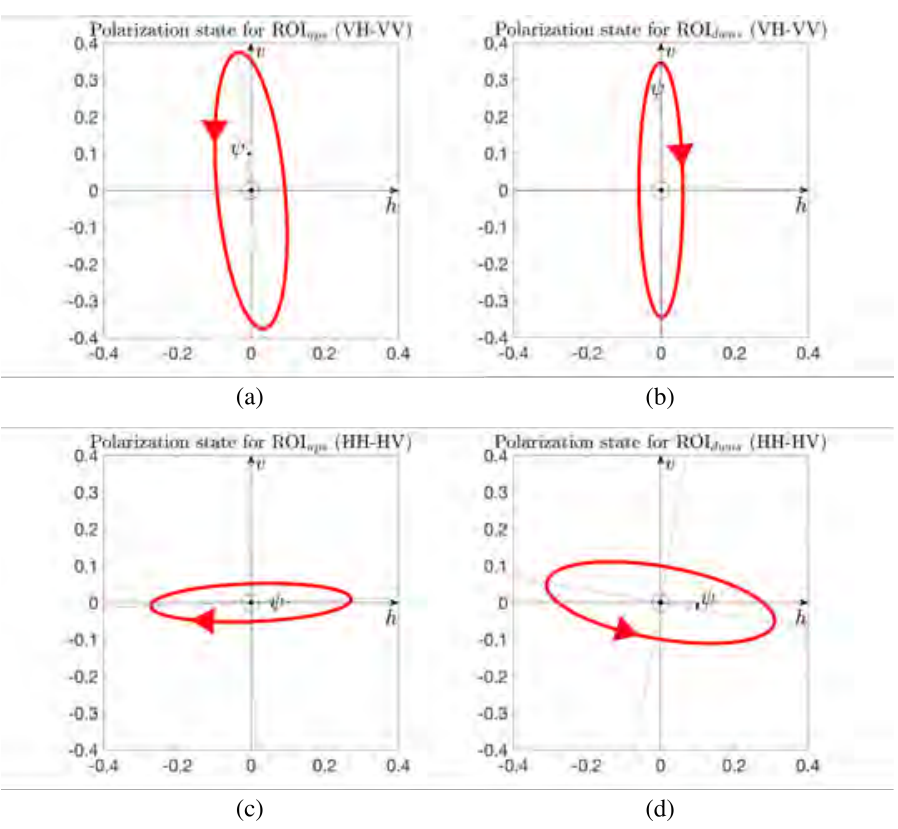

Fig. 12. Polarization state of the polarized term relevant to $\mathrm{ROI}_{u p s}$ and $\mathrm{ROI}_{d w n s}$. First row: VH-VV case; second row: HH-HV case.

We have developed two angles for the polarized component, $\alpha$ and $\delta$, that measure the transformation of polarization state on reflection. We note that these are not the same parameters as estimated from quad-pol systems, relating more to reflected wave transformations that are common in S1 data. The polarization state backscattered from the lower layer is not usually the same as the transmitted one, and can therefore be used for key applications, e.g., classification and geo-physical parameter estimation.

We have shown that the wave alpha angle exhibits important changes with time, especially for rice crops, and how this is linked to the vertical orientation of plants causing differential wave extinction during key phenology phases. This result reinforces the need for dense time series availability in future dualpol sensors. Importantly, our decomposition approach allows separation of the volume and the polarized contribution to the backscattered powers, showing that depolarization is not the only contribution to the cross-polarized intensity, as commonly assumed in the literature. The polarized contribution, which depends on $\alpha$, plays a key role which, for instance, allows decoupling the above-mentioned crop orientation effects from volume scattering in the canopy. One consequence of this is that the cross-polar channel ( $\mathrm{VH}$ or $\mathrm{HV}$ ) itself becomes a poor estimate of volume scattering which, if uncorrected, can lead, for example, to RVI values which exceed unity. Our decomposition approach always guarantees such radar vegetation indices remain bounded below 1 . This makes them better suited for fusion with optical systems employing NDVI for example.

The polarimetric phase, $\delta$, while largely zero for many land surfaces and noisy for forested terrain at C-band, can show important coherent spatial variations for certain applications. Key amongst these is one demonstrated here, the monitoring of land-ice, where we have shown that coherent dual-pol systems show strong phase signatures in glacial transition zones, i.e., around the edges of the glacier, where land-ice slopes occur. Here, the polarized term drastically changes the polarization state, resulting in elliptical polarization on receive. As such transition zones are key for studying glacier dynamics, we propose that dual-pol phase analysis coupled to dense time series would be a fruitful area of future study.

\section{APPENDIX A}

\section{MAPPING BETWEEN POLARIZATION STATES AND SCATTERING MECHANISMS}

To explore the relationship between polarization states and scattering mechanisms, it is necessary to relate the $\alpha$ angle to the scattering characteristics of the targets. To do this, we consider different values of the scattering angle $\alpha_{s}$ in (11) and obtain, for each of these values, several Stokes vectors by varying $\theta$ from $0^{\circ}$ to $90^{\circ}$. Then, from each Stokes vector we calculate $\alpha$ using (22). As a result, for a given value of $\alpha_{s}$ we obtain a curve relating $\alpha$ to $\theta$. Fig. 13 shows, for both the $\mathrm{H}$ and the $\mathrm{V}$ transmit case (where $\alpha$ is denoted with $\alpha_{H H H V}$ and $\alpha_{V H V V}$, respectively), the $\alpha / \theta$ curves for two ranges of $\alpha_{s}: \alpha_{s}<45^{\circ}$ (shown in Fig. 13(a)-(b)), where we highlight with the corresponding colors and circle marks the curves associated with $\alpha_{s}=0^{\circ}, \alpha_{s}=7.5^{\circ}, \alpha_{s}=22.5^{\circ}$, and $\alpha_{s}=37.5^{\circ} ; \alpha_{s}>45^{\circ}$ (shown in Fig. 13(c)-(d)), where we highlight with the corresponding colors and circle marks the curves associated with $\alpha_{s}=52.5^{\circ}, \alpha_{s}=67.5^{\circ}$, $\alpha_{s}=82.5^{\circ}$, and $\alpha_{s}=90^{\circ}$. Note that, in these plots, the curve for $\alpha_{s}=45^{\circ}$ is also shown with star marks. We clearly see that the $\alpha_{s} / \alpha$ mapping is ambiguous. In fact, a single scattering mechanism (that is $\alpha_{s}$ fixed) is mapped into many polarization states, depending on the value of the $\theta$ angle. We note another ambiguity for $\alpha_{s} \neq 45^{\circ}$ (the case of $\alpha_{s}=45^{\circ}$ is discussed afterward), where a given $\theta$ value of corresponds to several scattering mechanisms, and hence, to several polarization states. Furthermore, for a given $\alpha$ value, we observe ambiguities between the polarization states associated with $\alpha_{s}<45^{\circ}$ and the ones associated with $\alpha_{s}>45^{\circ}$. For instance, it is not possible to distinguish pure surface scattering $\left(\alpha_{s}=0^{\circ}, \theta=0^{\circ}\right)$ from dihedral scattering when the latter has $\theta=90^{\circ}$. On the other hand, if dihedral targets are characterized by $\theta=45^{\circ}$, this would result in larger $\alpha$ values with respect the pure surface scattering case. Only in such a situation $\alpha$ would provide a separation (up to a certain extent) of these two types of targets.

The above ambiguities are solved if both $\alpha_{s}$ and $\theta$ are known. When dealing with dual-pol SAR data, there is no way to determine these two parameters from $\underline{s}$ (2). Hence, this demonstrates that: (a) it is not possible to relate a single scattering mechanism to a given polarization state; (b) canonical scattering mechanisms, such as surface and dihedral, cannot be easily distinguished.

Let us now analyze the behavior of $\alpha$ for $\alpha_{s}=45^{\circ}$ (dipole scattering). In such a case, the curve which relates $\alpha$ to $\theta$ is the bisectrix of $\alpha / \theta$ plane (see Fig. 13). Therefore, it is now possible to unambiguously relate $\alpha$ to $\theta$. In particular, by 


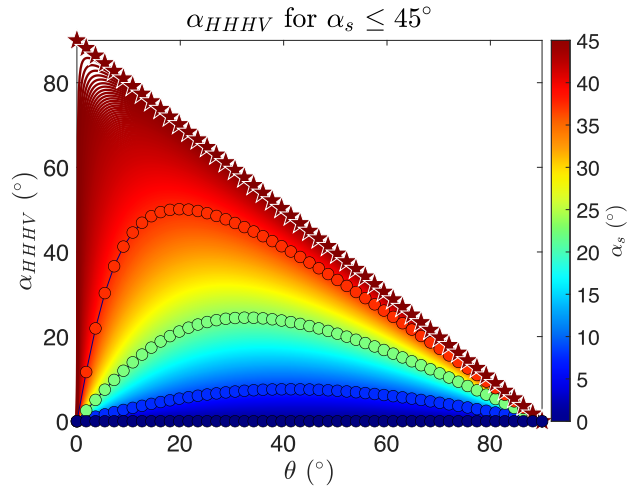

(a)

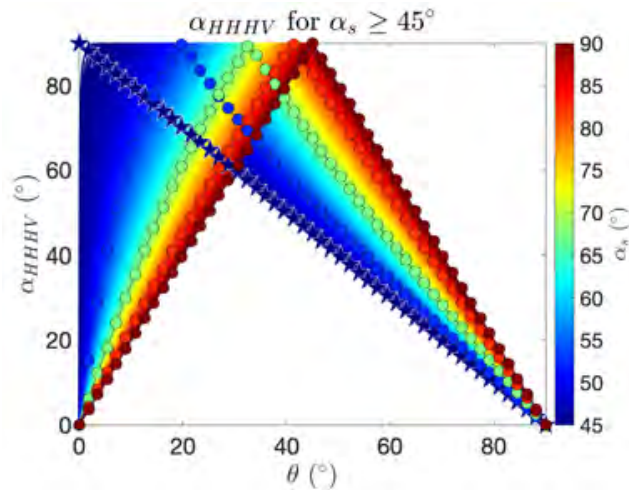

(c)

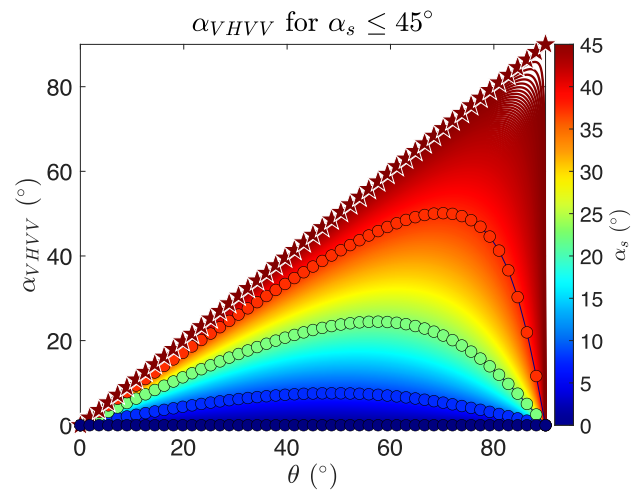

(b)

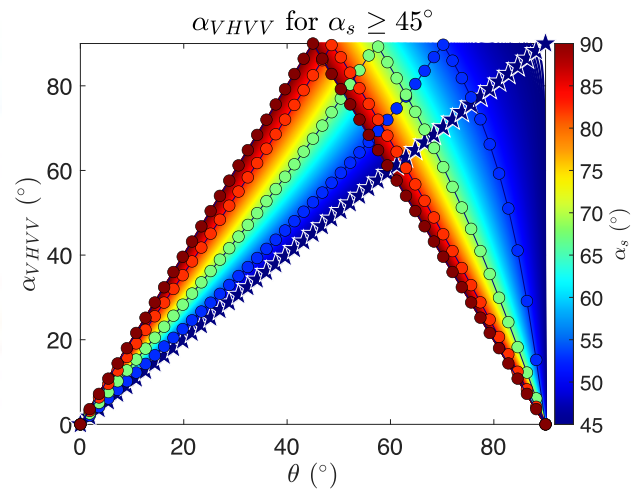

(d)

Fig. 13. $\alpha / \theta$ curves for different $\alpha_{s}$ values. First row: curves for $\alpha_{s} \leq 45^{\circ}$, with (a) and (b) showing Hand the $\mathrm{V}$ transmit case, respectively. The curve for $\alpha_{s}=45^{\circ}$ is shown with star marks, while circle marks represent the curves associated with $\alpha_{s}=0^{\circ}, \alpha_{s}=7.5^{\circ}, \alpha_{s}=22.5^{\circ}$ and $\alpha_{s}=37.5^{\circ}$. Second row: curves for $\alpha_{s} \geq 45^{\circ}$, with (c) and (d) showing $\mathrm{H}$ and the $\mathrm{V}$ transmit case, respectively. The curve for $\alpha_{s}=45^{\circ}$ is shown with star marks, while circle marks represent the curves associated with $\alpha_{s}=52.5^{\circ}, \alpha_{s}=67.5^{\circ}, \alpha_{s}=82.5^{\circ}$ and $\alpha_{s}=90^{\circ}$.

TABLE VII

RANGES OF $\alpha$ VALUES, FOR BOTH THE H AND THE V TRANSMIT CASE, ASSOCIATED WITH DIFFERENT TYPES OF DIPOLES AT VARIANCE OF $\theta\left(\alpha_{s}=45^{\circ}\right)$.

\begin{tabular}{|c|c|c|c|}
\hline $\begin{array}{c}\text { Horizontal } \\
\left(0^{\circ} \leq \theta \leq 22.5^{\circ}\right)\end{array}$ & $\begin{array}{c}\text { Almost horizontal } \\
\left(22.5^{\circ}<\theta \leq 45^{\circ}\right)\end{array}$ & $\begin{array}{c}\text { Almost vertical } \\
\left(45^{\circ}<\theta<67.5^{\circ}\right)\end{array}$ & $\begin{array}{c}\text { Vertical } \\
\left(67.5^{\circ} \leq \theta \leq 90^{\circ}\right)\end{array}$ \\
\hline $67.5^{\circ}<\alpha_{H H H V} \leq 90^{\circ}$ & $45^{\circ} \leq \alpha_{H H H V} \leq 67.5^{\circ}$ & $22.5^{\circ} \leq \alpha_{H H H V}<45^{\circ}$ & $0^{\circ} \leq \alpha_{H H H V} \leq 22.5^{\circ}$ \\
\hline $0^{\circ}<\alpha_{V H V V}<22.5^{\circ}$ & $22.5^{\circ} \leq \alpha_{V H V V} \leq 45^{\circ}$ & $45^{\circ}<\alpha_{V H V V} \leq 67.5^{\circ}$ & $67.5^{\circ}<\alpha_{V H V V} \leq 90^{\circ}$ \\
\hline
\end{tabular}

setting $\alpha_{s}=45^{\circ}$ in (11), we consider the following important cases:

- $\boldsymbol{\theta}=\mathbf{0}^{\circ}$, horizontal dipole. The horizontal polarization is perfectly aligned with the symmetry axis of the dipole, while the vertical one has a $90^{\circ}$ tilt. Therefore, $\mathrm{H}(\mathrm{V})$ polarized waves are completely absorbed (maintained) with $\underline{s}$ being $\underline{s}=\left[\begin{array}{llll}0, & 0,0,0\end{array}\right]^{T}\left(\underline{s}=\left[\begin{array}{lll}1,-1, & 0,0\end{array}\right]^{T}\right)$. Accordingly, $\alpha_{H H H V}=90^{\circ}$ and $\alpha_{V H V V}=0^{\circ}$.

- $\boldsymbol{\theta}=22.5^{\circ}$. In this case, $\underline{s}=[1 \mp \sqrt{2} / 2,-\sqrt{2} / 2 \pm$ $0.5,-\sqrt{2} / 2 \pm 0.5,0]^{T}$. Accordingly, $\alpha_{H H H V}=67.5^{\circ}$ and $\alpha_{V H V V}=22.5^{\circ}$.

- $\boldsymbol{\theta}=45^{\circ}$. In this case, the $\mathrm{H}$ and the $\mathrm{V}$ polarizations have equal amplitudes resulting in $\underline{s}=\left[\begin{array}{ll}1,0,-1.0 & 0\end{array}\right]^{T}$, which corresponds to a $-45^{\circ}$ polarization. Accordingly, $\alpha_{H H H V}=\alpha_{V H V V}=45^{\circ}$.

- $\boldsymbol{\theta}=67.5^{\circ}$. In this case, $\underline{s}=[1 \pm \sqrt{2} / 2, \sqrt{2} / 2 \pm$ $0.5,-\sqrt{2} / 2 \mp 0.5,0]^{T}$, for which $\alpha_{H H H V}=22.5^{\circ}$ and $\alpha_{V H V V}=67.5^{\circ}$.
- $\boldsymbol{\theta}=90^{\circ}$, vertical dipole. The vertical polarization is perfectly aligned with the symmetry axis of the dipole, while the horizontal one has a $90^{\circ}$ tilt. Hence, V (H) polarized waves are completely absorbed (maintained) with $\underline{s}$ being $\underline{s}=\left[\begin{array}{lll}0,0, & 0,0\end{array}\right]^{T}\left(\underline{s}=\left[\begin{array}{lll}1, & 1,0,0\end{array}\right]^{T}\right)$. Accordingly, $\alpha_{H H H V}=0^{\circ}$ and $\alpha_{V H V V}=90^{\circ}$.

The above cases allow us defining different ranges of $\alpha_{H H H V}$ and $\alpha_{V H V V}$, which can be associated with the scattering from different types of dipoles, as shown in Table VII. Note that this table can be used if we have an (approximate) a priori knowledge of target that is being observed, for which we are confident that the dipole scattering assumption (widely employed in radar remote sensing) holds. In such a case we do not need to know the specific $\theta$ values, as its ranges can be deduced from Table VII, once $\alpha$ is estimated from the observed Stokes vector using (22). For instance, if we are observing the sea surface at high incidence angle, the $\mathrm{H}$ polarization is significantly attenuated with respect to the $\mathrm{V}$ one, which 
can be seen as the scattering from a horizontal dipole, where $67.5^{\circ}<\alpha_{H H H V}\left(\alpha_{V H V V}<22.5^{\circ}\right)$ is expected.

Another important example is dipole scattering from vegetation. Here, we can distinguish between two cases: weakly oriented and strongly oriented dipoles. In the first case, when the scatterers (leaves, stems, branches, etc.) do not have a predominant orientation, $\alpha_{H H H V}$ and $\alpha_{V H V V}$ are expected to vary in the range $22.5^{\circ}-67.5^{\circ}$, which embraces the "Almost horizontal" and "Almost vertical" cases (see Table VII). This corresponds, for example, to the scattering from longer (in terms of wavelength) tree branches, and the scattering from thin narrow leaves in non cereal crops. Note that the limiting case for such weakly oriented scatterers is $\alpha_{H H H V}=\alpha_{V H V V}=45^{\circ}$, for which vertical and polarized waves have equal amplitudes. The strongly oriented dipoles class includes, for instance, cereal crops (e.g., rice) in the stem elongation stages, where the vertical stems induce a significant attenuation on the $\mathrm{V}$ polarized waves. These belong to the "Almost vertical" and "Vertical" cases in Table VII, for which $45^{\circ}<\alpha_{V H V V}$ and $\alpha_{H H H V}<22.5^{\circ}$ are expected.

Regarding other types of crops, such as broad leaves crops, we consider corn plants as an example. We expect that the dipole assumption does not hold in this case, since corn leaves have large size compared to the wavelength. As a consequence, according also to the analysis in [28], we expect a strong surface scattering contribution due to: (a) the spacing between the corn plants at earliest growth stages; (b) direct scattering from the large leaves as plants develop. Accordingly, we expect $\alpha_{s}<45^{\circ}$ and $\theta<45^{\circ}$ (surface scattering) and thus both $\alpha_{H H H V}$ and $\alpha_{V H V V}$ to be low.

\section{ACKNOWLEDGMENT}

RADARSAT-2 Data and Products CMacDonald, Dettwiler and Associates Ltd. (MDA) (2014)—All Rights Reserved. RADARSAT is an official trademark of the Canadian Space Agency (CSA). All RADARSAT-2 images were provided by MDA and CSA in the framework of project SOAR-EI 5158.

\section{REFERENCES}

[1] I. Hajnsek and Y. L. Desnos, Eds., Polarimetric Synthetic Aperture Radar. Principles and Application. Springer International Publishing, 2021.

[2] J. Lee and E. Pottier, Polarimetric Radar Imaging: From Basics to Applications. Boca Raton, FL, USA: CRC Press, 2009.

[3] S. R. Cloude, "Radar target decomposition theorems," IEE Electronics Letters, vol. 21, no. 1, pp. 22-24, January 1985.

[4] S. R. Cloude and E. Pottier, "A review of target decomposition theorems in radar polarimetry," IEEE Trans. Geosci. Remote Sensing, vol. 34, no. 2, pp. 498-518, March 1996.

[5] S. R. Cloude, Polarisation: Applications in Remote Sensing. Oxford University Press, 978-0-19-956973-1, 2009.

[6] A. Freeman and T. Durden, "A three-component scattering model for polarimetric SAR data," IEEE Trans. Geosci. Remote Sensing, vol. 36, no. 3, pp. 963-973, May 1998.

[7] Y. Yamaguchi, T. Moriyama, M. Ishido, and H. Yamada, "Fourcomponent scattering model for polarimetric SAR image decomposition," IEEE Trans. Geosci. Remote Sensing, vol. 43, no. 8, pp. 16991706, August 2005

[8] W. T. An, Y. Cui, and J. Yang, "Three-component model-based decomposition for polarimetric SAR data," IEEE Trans. Geosci. Remote Sensing, vol. 48, no. 6, pp. 2732-2739, 2010.

[9] S. W. Chen, X. Wang, S. Xiao, and M. Sato, "General polarimetric model- based decomposition for coherency matrix," IEEE Trans. Geosci. Remote Sensing, vol. 52, no. 3, pp. 1843-1855, 2014.
[10] Q. Xie, J. D. Ballester-Berman, J. Lopez-Sanchez, Z. Jianjun, and W. Changcheng, "Quantitative analysis of polarimetric model-based decomposition methods," Remote Sens., vol. 8, no. 12, p. 977, 2016.

[11] T. L. Ainsworth, Y. Wang, and J. S. Lee, "Model-based polarimetric SAR decomposition: An $L_{1}$ regularization approach," IEEE Trans. Geosci. Remote Sensing, 2021, DOI: 10.1109/TGRS.2021.3083511.

[12] R. Touzi, "A review of speckle filtering in the context of estimation theory," IEEE Trans. Geosci. Remote Sensing, vol. 40, no. 11, pp. 2392 2404, 2002.

[13] S. R. Cloude, D. Goodenough, and H. Chen, "Compact decomposition theory," IEEE Geosci. Remote Sens. Lett., vol. 9, no. 1, pp. 28-32, 2012.

[14] S. Cloude, D. Goodenough, H. Chen, Y. Rao, and W. Hong, "Pauli phase calibration in compact polarimetry," IEEE J. Sel. Topics Appl. Earth Observations Remote Sens., vol. 11, no. 12, pp. 4906-4917, December 2018.

[15] J. M. Lopez-Sanchez, F. Vicente-Guijalba, J. Ballester-Berman, and S. Cloude, "Polarimetric response of rice fields at C-band: Analysis and phenology retrieval," IEEE Trans. Geosci. Remote Sensing, vol. 52, no. 5, pp. 2977-2993, 2014.

[16] J. M. Lopez-Sanchez, S. R. Cloude, and J. D. Ballester-Berman, "Rice phenology monitoring by means of SAR polarimetry at X-band," IEEE Trans. Geosci. Remote Sensing, vol. 50, no. 7, pp. 2695-2709, 2012.

[17] L.Mascolo, J.M.Lopez-Sanchez, and S. Cloude, "Thermal noise removal from polarimetric Sentinel-1 data,' IEEE Geosci. Remote Sens. Lett., 2021, DOI: 10.1109/LGRS.2021.3050921.

[18] J. J. V. Zyl, M. Arii, and Y. Kim, "Model-based decomposition of polarimetric SAR covariance matrices constrained for nonnega- tive eigenvalues,", IEEE Trans. Geosci. Remote Sensing, vol. 49, no. 9, pp. 3452-3459, September 2011.

[19] M. Li and W. Bijker, "Potential of multi-temporal Sentinel-1A dual polarization SAR images for vegetable classification in indonesia," in IGARSS 2018 - 2018 IEEE International Geoscience and Remote Sensing Symposium, vol. 2, 2018, pp. 3829-3823, dOI: 10.1109/IGARSS.2018.8517325.

[20] X. Banque, J. M. Lopez-Sanchez, D. Monells, J. D. Ballester-Berman, J. Duro, and F. Koudogbo, "Polarimetry-based land cover classification with Sentinel-1 data," in Proceedings of the 7th International Workshop on Science and Applications of SAR Polarimetry and Polarimetric Interferometry (POLinSAR'2015), vol. SP-729, Frascati, Italy, January 2015.

[21] K. Harfenmeister, S. Itzerott, C. Weltzien, and D. Spengler, "Agricultural monitoring using polarimetric decomposition parameters of Sentinel-1,' Remote Sens., vol. 13, no. 4, p. 575, 2021.

[22] D. Mandal, V. Kumar, D. Ratha, S. Dey, A. Bhattacharya, J. M. LopezSanchez, H. McNairn, and Y. S. Rao, "Dual polarimetric radar vegetation index for crop growth monitoring using Sentinel-1 SAR data," Remote Sensing of Environment, vol. 247, no. 111954, September 2020.

[23] A. Mestre-Quereda, J. M. Lopez-Sanchez, F. Vicente-Guijalba, A. W. Jacob, and M. E. Engdahl, "Time-series of Sentinel-1 interferometric coherence and backscatter for crop-type mapping," IEEE J. Sel. Topics Appl. Earth Observations Remote Sens., vol. 13, pp. 4070-4084, 2020.

[24] G. Parrella, I. Hajnsek, and K. P. Papathanassiou, "Polarimetric decomposition of L-band PolSAR backscattering over the Austfonna ice cap," IEEE Trans. Geosci. Remote Sensing, vol. 54, no. 3, pp. 1267-1281, 2016.

[25] J. A. Dowdeswell, D. J. Drewry, A. P. R. Cooper, M. R. Gorman, O. Liestøl, and O. Orheim, "Digital Mapping of the Nordaustlandet Ice Caps from Airborne Geophysical Investigations," Annals of Glaciology, vol. 8, pp. 51-58, 1986.

[26] G. Parrella, I. Hajnsek, and K. P. Papathanassiou, "Model-Based Interpretation of PolSAR Data for the Characterization of Glacier Zones in Greenland," IEEE J. Sel. Topics Appl. Earth Observations Remote Sens., 2021, DOI: 10.1109/JSTARS.2021.3126069.

[27] M. Schäfer, T. Zwinger, P. Christoffersen, , F. Gillet-Chaulet, K. Laakso, R. Pettersson, V. Pohjola, T. Strozzi, and J. C. Moore, "Sensitivity of basal conditions in an inverse model: Vestfonna ice cap, Nordaustlandet/Svalbard," The Cryosphere, vol. 6, no. 4, pp. 771-783, 2012.

[28] C. Liu, J. Shang, P. W. Vachon, and H. McNairn, "Multiyear Crop Monitoring Using Polarimetric RADARSAT-2 Data," IEEE Trans. Geosci. Remote Sensing, vol. 51, no. 4, pp. 2227-2240, 2013. 


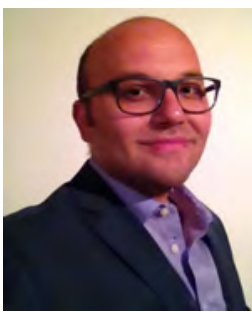

Lucio Mascolo (S'12-M'16) was born in Italy in 1983. He received the B.Sc. and M.Sc. degrees (summa cum laude) in telecommunications engineering from the Universita' degli Studi di Napoli Parthenope, Naples, Italy, in 2007 and 2011, respectively, and the Ph.D. degree in electronic and information engineering (curriculum electromagnetic fields) from the Universita' degli Studi di Cagliari, Cagliari, Italy, in 2015. From 2015 to 2016, he was a Postdoctoral Fellow with the Electromagnetics and Remote Sensing Group, Universita' degli Studi di Napoli Parthenope. From 2016 to 2018, he was a Researcher with SarVision, Wageningen, The Netherlands, and from 2019 to 2020, he was with Digital Content Analysis Technology (DCAT), Glasgow, U.K. In 2020 he joined the Signals, Systems and Telecommunication at University of Alicante, Spain, where his research activities include electromagnetic modeling, radar polarimetry, polarimetric SAR data analysis, and remote sensing for land applications.

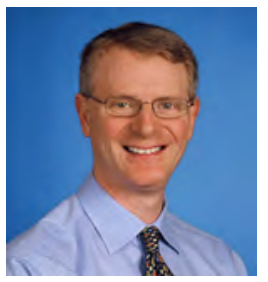

Shane R. Cloude received the B.Sc. (Hons.) degree from the University of Dundee, U.K., in 1981, and the Ph.D. degree from the University of Birmingham, U.K., in 1987. He was then a Radar Scientist with the Royal Signals and Radar Establishment, Great Malvern, U.K. Following this, he held teaching and research posts at the University of Dundee, U.K., the University of York, U.K. and the University of Nantes, France, before taking on his present role in 2001. He is now Senior Scientist with AEL Consultants, undertaking research on a range of topics associated with radar and optics. His main research interests are in polarization effects in electromagnetic scattering and their applications in radar and optical remote sensing. He is the author of 2 books, 10 book chapters, 53 journal publications, and over 180 international conference and workshop papers. Dr. Cloude is a Fellow of the Alexander von Humboldt Foundation in Germany and has held Honorary Professorships and Chairs at the Universities of Dundee and Australia. York, UK and the University of Adelaide, Australia.

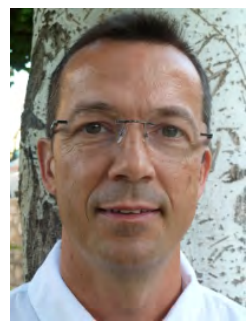

Juan M. Lopez-Sanchez (Senior Member, IEEE) was born in Alicante, Spain, in 1972. He received the Ingeniero (M.S.) and Doctor Ingeniero (Ph.D.) degrees in telecommunication engineering from the Technical University of Valencia (UPV), Valencia, Spain, in 1996 and 2000, respectively. From 1998 to 1999, he worked as a Predoctoral Grantholder with the Space Applications Institute, Joint Research Centre of the European Commission, Ispra, Italy. Since 2000, he leads the Signals, Systems and Telecommunication Group of the University of Alicante, Spain, where he is a Full Professor since November 2011. His research interests include microwave remote sensing for inversion of biophysical parameters, polarimetric and interferometric techniques, SAR imaging algorithms, and applications of radar remote sensing in agriculture and geophysics. He has coauthored more than 90 papers in refereed journals and more than 140 papers and presentations in international conferences and symposia. Dr. LopezSanchez received the Indra Award for the best Ph.D. thesis about radar in Spain, in 2001. From 2006 to 2012, he was the Chair of the Spanish Chapter of the IEEE Geoscience and Remote Sensing Society. 